\title{
ASYMPTOTIC FORMULAS \\ FOR TRAPPED MODES AND FOR EIGENVALUES \\ BELOW THE THRESHOLD OF THE CONTINUOUS SPECTRUM OF A WAVEGUIDE WITH A THIN SCREENING BARRIER
}

\author{
S. A. NAZAROV
}

\begin{abstract}
Asymptotic formulas are found for the eigenvalues and eigenfunctions (trapped modes) of the mixed boundary value problem for the Laplace operator in an $n$-dimensional cylindrical waveguide with a thin screening barrier obtained by a regular perturbation of a part $\theta$ of the hyperplane orthogonal to the axis of the cylinder; the boundary $\partial \theta$ is smooth and $(n-1)$-dimensional. These asymptotic formulas agree with the sufficient conditions for the discrete spectrum to be nonempty, deduced via the variational method. For an unbounded waveguide, both the algorithm for obtaining asymptotic formulas, and even the orders themselves of the main correction terms turn out to be different from those for a bounded domain. The same refers to the justification procedure for asymptotic expansions, which employs substantially the spectral theory machinery.
\end{abstract}

\section{§1. INTRODUCTION}

1. Waveguide with screen. Let $\omega$ and $\theta \subset \omega$ be domains in the Euclidean space $\mathbb{R}^{n-1}$, with smooth boundaries $\partial \omega$ and $\partial \theta$ and compact closures $\bar{\omega}=\omega \cup \partial \omega$ and $\bar{\theta}$, and let $\bar{\theta} \subset \omega$. Also, let $\Omega=\omega \times \mathbb{R} \ni(y, z)$ be a cylinder (waveguide). Suppose that the closed set (a thin screening barrier)

$$
\Theta^{\varepsilon}=\left\{x=\left(x_{1}, \ldots, x_{n-1}, x_{n}\right)=(y, z): y \in \bar{\theta},-\varepsilon H_{-}(y) \leq z \leq \varepsilon H_{+}(y)\right\},
$$

see Figure 1a or Figure 1b, depends on a small positive parameter $\varepsilon$ and is defined in (1.1) with the help of two functions $H_{ \pm}$smooth in $\bar{\theta}$ and such that

$$
\begin{aligned}
H(y) & :=H_{-}(y)+H_{+}(y) \geq 0, \quad y \in \theta, \\
H_{ \pm}(y) & =0, \quad y \in \partial \theta .
\end{aligned}
$$

The first requirement imposed on the screen thickness function $H$ is natural and clear, but the second is caused by technical difficulties (see below the calculations and discussion in Subsection 4 of $\S 2$ ) and means that the screen $\Theta^{\varepsilon}$ has a sharp edge $\vartheta^{\varepsilon}$ (an edge with zero opening) in the hyperplane $\{x: z=0\}$, which splits the boundary $\partial \Theta^{\varepsilon}$ into two smooth surfaces:

$$
\theta_{ \pm}^{\varepsilon}=\left\{(y, z): y \in \theta, z= \pm \varepsilon H_{ \pm}(y)\right\} .
$$

The smoothness requirements on the profile functions $H_{ \pm}$and the boundary $\partial \theta$ (unlike the boundary $\partial \omega$ ) are also inherent. In the domain $\Omega^{\varepsilon}=\Omega \backslash \Theta^{\varepsilon}$ (waveguide with barrier;

2010 Mathematics Subject Classification. Primary 35J05.

Key words and phrases. Waveguide with barrier, trapped modes, discrete and continuous spectra, asymptotic formulas for eigenvalues, boundary perturbation, spectral measure.

Supported by RFBR (grant no. 09-01-00759). 
a

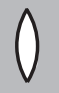

$\mathrm{b}$

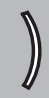

FiguRE 1. Waveguide with screen.

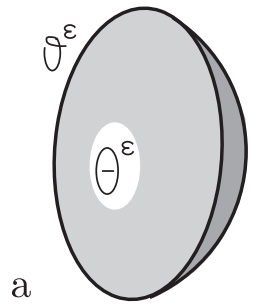

$\mathrm{b}$

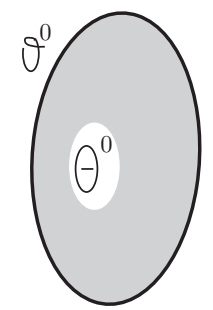

FiguRE 2. Screens: curved (a) and straight (b).

see Figure 1) we consider the following mixed boundary value problem for the Helmholtz equation:

$$
\begin{aligned}
-\Delta_{x} u^{\varepsilon}(x) & =\lambda^{\varepsilon} u^{\varepsilon}(x), \quad x \in \Omega^{\varepsilon}, \\
u^{\varepsilon}(x) & =0, \quad x \in \Gamma=\partial \Omega, \\
\partial_{\nu} u^{\varepsilon}(x) & =0, \quad x \in \theta_{ \pm}^{\varepsilon} .
\end{aligned}
$$

Here $\Delta_{x}$ is the Laplace operator written in the Cartesian coordinates $x, \lambda^{\varepsilon}$ is the spectral parameter, and $\partial_{\nu}$ stands for the derivative along the outward normal. The variational statement of problem (1.5)-(1.7) reduces to the integral identity

$$
\left(\nabla_{x} u^{\varepsilon}, \nabla_{x} v^{\varepsilon}\right)_{\Omega^{\varepsilon}}=\lambda^{\varepsilon}\left(u^{\varepsilon}, v^{\varepsilon}\right)_{\Omega^{\varepsilon}}, \quad v^{\varepsilon} \in \stackrel{\circ}{H}^{1}\left(\Omega^{\varepsilon} ; \Gamma\right)
$$

(see [1]), where $\nabla_{x}$ is the gradient operator, $(,)_{\Omega^{\varepsilon}}$ is the scalar product in the Lebesgue space $L_{2}\left(\Omega^{\varepsilon}\right)$, and $\stackrel{\circ}{H}^{1}\left(\Omega^{\varepsilon} ; \Gamma\right)$ is the space of all functions in the Sobolev class $H^{1}\left(\Omega^{\varepsilon}\right)$ that satisfy the Dirichlet condition (1.6).

If $\varepsilon=0$, the set (1.1) turns into a part of the $(n-1)$-dimensional hyperplane $\{x$ : $z=0\}$, becoming a straight screen of zero thickness (Figure $2 \mathrm{~b}$ )

$$
\Theta^{0}=\{x: y \in \bar{\theta}, z=0\} .
$$

Note that only connected sets (1.9) and (1.1) are treated in $\S \S 2$ and 3, but simple generalizations to the case of several connected components of the sets $\Theta^{0}$ and $\Theta^{\varepsilon}$ are mentioned in Subsection 1 of $\S 4$. The set (1.1) itself will be called a curved screen; it has zero thickness if $H_{+}=-H_{-}$(the thickness function (1.2) vanishes everywhere on $\theta$ ).

The auxiliary Dirichlet problem on the cross section, namely, the problem

$$
-\Delta_{y} U(y)=\Lambda U(y), \quad y \in \omega, \quad U(y)=0, \quad y \in \partial \omega,
$$

has discrete spectrum. Its first eigenvalue $\Lambda_{\dagger}=\Lambda_{1}$ is positive and simple, and the corresponding eigenfunction $U_{\dagger}=U_{1}$ can be taken positive in $\omega$ (see, e.g., [2]). In what 
follows, we assume that $U_{\dagger}$ is normalized in $L_{2}(\omega)$, i.e.,

$$
\left\|U_{\dagger} ; L_{2}(\omega)\right\|=1, \quad \text { whence, } \quad\left\|\nabla_{y} U_{\dagger} ; L_{2}(\omega)\right\|=\sqrt{\Lambda_{\dagger}} .
$$

Due to the cylindrical structure of $\Omega^{\varepsilon}$, near infinity for any function $v^{\varepsilon} \in \stackrel{\circ}{H}^{1}\left(\Omega^{\varepsilon} ; \Gamma\right)$ we have

$$
\left\|v^{\varepsilon} ; L_{2}\left(\Omega^{\varepsilon}\right)\right\| \leq c\left\|\nabla_{x} v^{\varepsilon} ; L_{2}\left(\Omega^{\varepsilon}\right)\right\| .
$$

Observe that, by the definition (1.1) and the requirements imposed on $\theta, \omega$, and $H_{ \pm}$, the factor $c$ in (1.12) can be fixed to serve all $\varepsilon \in(0,1]$ (see Remark 3.2).

Inequality (1.12) allows us to view the left-hand side of (1.8) as a scalar product in the Hilbert space $\stackrel{\circ}{H}^{1}\left(\Omega^{\varepsilon} ; \Gamma\right)$. Thus, the quadratic form $\left(\nabla_{x} u^{\varepsilon}, \nabla_{x} u^{\varepsilon}\right)_{\Omega^{\varepsilon}}$ is closed and lower semibounded on $L_{2}\left(\Omega^{\varepsilon}\right)$, so that the variational problem (1.8) gives rise (see [3, Chapter 10]) to an unbounded positive selfadjoint operator $A^{\varepsilon}$ in $L_{2}\left(\Omega^{\varepsilon}\right)$. It is known that the spectrum $\sigma^{\varepsilon}$ consists (see, e.g., [4, 5]) of the continuous spectrum $\sigma_{c}^{\varepsilon}=\left[\Lambda_{\dagger},+\infty\right.$ ) and the possibly nonempty discrete spectrum $\sigma_{d}^{\varepsilon} \subset\left(0, \Lambda_{\dagger}\right)$. Our main goal in this paper is to find conditions ensuring that eigenvalues do emerge on $\left(0, \Lambda_{\dagger}\right)$, to compute the number of such eigenvalues, and to deduce asymptotic formulas as $\varepsilon \rightarrow+0$.

It should be emphasized that for the limiting $(\varepsilon=0)$ problem

$$
\begin{aligned}
-\Delta_{x} u^{0}(x) & =\lambda^{0} u^{0}(x), \quad x \in \Omega^{0}, \quad u^{0}(x)=0, \quad x \in \Gamma, \\
\partial_{\nu} u^{0}(x) & =0, \quad x \in \theta_{ \pm}^{0},
\end{aligned}
$$

where the $\theta_{ \pm}^{0}$ are the shores of the cut (1.9), the discrete spectrum is always empty, because integrating over $z$ of the Friedrichs inequality

$$
\Lambda_{\dagger}\left\|V ; L_{2}(\omega)\right\|^{2} \leq\left\|\nabla_{y} V ; L_{2}(\omega)\right\|^{2}, \quad V \in \stackrel{\circ}{H}^{1}(\omega ; \partial \omega),
$$

on the cut $\omega$ (a straight screen of zero thickness does not hamper this) yields the inequality

$$
\Lambda_{\dagger}\left\|v ; L_{2}\left(\Omega^{0}\right)\right\|^{2} \leq\left\|\nabla_{y} v ; L_{2}\left(\Omega^{0}\right)\right\|^{2}, \quad v \in \stackrel{\circ}{H}^{1}\left(\Omega^{0} ; \Gamma\right),
$$

which means that there is no spectrum $\sigma^{0}$ below the threshold $\Lambda_{+}$.

The following simple observation serves as a starting point for the asymptotic analysis done in $\S 2$ : problem (1.13), (1.14) with the parameter $\lambda=\Lambda_{\dagger}$ admits the solution

$$
w^{0}(x)=U_{\dagger}(y) .
$$

Indeed, identities (1.13) are implied by (1.10), and the Neumann boundary condition on the two surfaces $\theta_{ \pm}^{0}$ of the screen (1.9) is fulfilled because $\partial_{\nu}=\mp \partial_{z}=\mp \partial / \partial z$, but the function (1.17) does not depend on $z$.

In a free (without a screen) waveguide $\Omega$, besides (1.13), the Dirichlet problem (1.17) has yet another solution in the class of functions of polynomial growth as $z \rightarrow \pm \infty$, namely, the function

$$
z U_{\dagger}(y) \text {. }
$$

It turns out that problem (1.13), (1.14) also possesses a solution $w^{1}$ with a similar behavior at infinity (see Lemma 2.1). The formal asymptotic expressions of a possible eigenfunction $u_{1}^{\varepsilon}$ of problem (1.5)-(1.7) are sought in the form of a small perturbation of a linear combination of the solutions $w^{0}$ and $w^{1}$ (see Subsection 2 in $\S 2$ ); however, since the summands $w^{q}(x)$ do not decay properly at infinity, the initial asymptotic construction requires correction for large $|z|$ (see Subsection 3 in $\S 2$ ). There are two situations in which the construction of formal asymptotic expansions differ:

$$
\int_{\theta} H(y)\left(\left|\nabla_{y} U_{\dagger}(y)\right|^{2}-\Lambda_{\dagger}\left|U_{\dagger}(y)\right|^{2}\right) d y>0
$$


and

$$
\int_{\theta} H(y)\left(\left|\nabla_{y} U_{\dagger}(y)\right|^{2}-\Lambda_{\dagger}\left|U_{\dagger}(y)\right|^{2}\right) d y=0
$$

(see Subsections 2, 3, and 4 in $\S 2$ ). In any case, the final asymptotic formulas differ substantially (even in the order of the main correction term) from the asymptotic formulas for the solutions of spectral problems in bounded domains in the case of small perturbations of the boundary (see [3, Chapters 5, 6]).

Many geometrical restrictions are adopted in order to simplify the presentation; possible generalizations are discussed in $\S 4$.

2. Sufficient conditions for the existence of the discrete spectrum. In the papers [8, 9], a variational method was described for the search of eigenvalues below the continuous spectrum threshold. This method, collected from several sources, consists mainly of two technical and easily implementable tricks. For the reader's convenience, here we reproduce the corresponding calculations, for which the presence of the small parameter is irrelevant.

In accordance with [3, Chapter 10], the lower bound $\underline{\sigma}^{\varepsilon}$ of the operator $A^{\varepsilon}$ is calculated by the formula

$$
\underline{\sigma}^{\varepsilon}=\inf _{u^{\varepsilon} \in \dot{H}^{1}\left(\Omega^{\varepsilon} ; \Gamma\right)} \frac{\left\|\nabla_{x} u^{\varepsilon} ; L_{2}\left(\Omega^{\varepsilon}\right)\right\|^{2}}{\left\|u^{\varepsilon} ; L_{2}\left(\Omega^{\varepsilon}\right)\right\|^{2}} .
$$

It should be noted that the infimum in (1.19) is over the space $\stackrel{\circ}{H}^{1}\left(\Omega^{\varepsilon} ; \Gamma\right)$ occurring in (1.8), but not over the domain of $A^{\varepsilon}$, which is narrower; this is the advantage of the use of the results of [3], which reveal a bijection between the semibounded selfadjoint operators and the closed semibounded quadratic forms.

With the goal to arrive at a contradiction, suppose that the discrete spectrum is empty, i.e., $\underline{\sigma}^{\varepsilon}=\Lambda_{\dagger}$, by formula (1.19) and the unconditional identity $\sigma_{c}^{\varepsilon}=\left[\Lambda_{\dagger},+\infty\right)$. Hence, by (1.19) we have the estimate

$$
\Lambda_{\dagger}\left\|u^{\varepsilon} ; L_{2}\left(\Omega^{\varepsilon}\right)\right\|^{2} \leq\left\|\nabla_{x} u^{\varepsilon} ; L_{2}\left(\Omega^{\varepsilon}\right)\right\|^{2}, \quad u^{\varepsilon} \in \stackrel{\circ}{H^{1}}\left(\Omega^{\varepsilon} ; \Gamma\right),
$$

similar to (1.16). Here we plug the test function

$$
u^{\varepsilon \delta}(x)=\exp (-\delta|z|) U_{\dagger}(y),
$$

where $\delta$ is a small positive number. The decaying exponential factor ensures that $u^{\varepsilon \delta} \in$ $\stackrel{\circ}{H}^{1}\left(\Omega^{\varepsilon} ; \Gamma\right)$. We have

$$
\begin{aligned}
& \left\|u^{\varepsilon \delta} ; L_{2}\left(\Omega^{\varepsilon}\right)\right\|^{2}=\left\|u^{\varepsilon \delta} ; L_{2}(\Omega)\right\|^{2}-\left\|u^{\varepsilon \delta} ; L_{2}\left(\Theta^{\varepsilon}\right)\right\|^{2} \\
& \quad=\int_{-\infty}^{+\infty} \exp (-2 \delta|z|) d z\left\|U_{\dagger} ; L_{2}(\omega)\right\|^{2}-\int_{\Theta^{\varepsilon}} \exp (-2 \delta|z|)\left|U_{\dagger}(y)\right|^{2} d x \\
& \quad=\frac{1}{\delta}-\int_{\Theta^{\varepsilon}}\left|U_{\dagger}(y)\right|^{2} d x+O(\delta), \\
& \left\|\nabla_{x} u^{\varepsilon \delta} ; L_{2}\left(\Omega^{\varepsilon}\right)\right\|^{2}=\frac{1}{\delta}\left\|\nabla_{y} U_{\dagger} ; L_{2}(\omega)\right\|^{2}+\delta^{2} \int_{-\infty}^{+\infty} \exp (-2 \delta|z|) d z\left\|U_{\dagger} ; L_{2}(\omega)\right\|^{2} \\
& \quad-\int_{\Theta^{\varepsilon}} \exp (-2 \delta|z|)\left|\nabla_{x} u^{\varepsilon \delta}(x)\right|^{2} d x=\frac{\Lambda_{\dagger}}{\delta}-\int_{\Theta^{\varepsilon}}\left|\nabla_{y} U_{\dagger}(y)\right|^{2} d x+O(\delta) .
\end{aligned}
$$

We have used (1.11). Substituting the above formulas in (1.20), we see that the terms of order of $\delta^{-1}$ mutually cancel; the resulting inequality takes the form

$$
\int_{\Theta^{\varepsilon}}\left(\left|\nabla_{y} U_{\dagger}(y)\right|^{2}-\Lambda_{\dagger}\left|U_{\dagger}(y)\right|^{2}\right) d x \leq c \delta .
$$


a

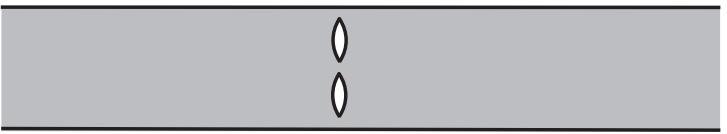

$\mathrm{b}$

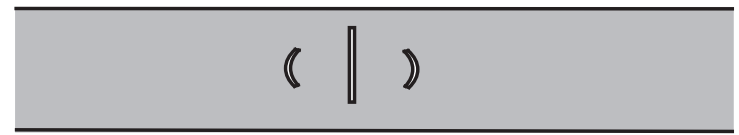

FiguRE 3. Waveguides with several screens.

If the integral on the left in (1.22) is positive, then the desired contradiction is reached for small $\delta$, i.e., the relation

$$
I_{\dagger}\left(U_{\dagger}\right):=\int_{\Theta^{\varepsilon}}\left(\left|\nabla_{y} U_{\dagger}(y)\right|^{2}-\Lambda_{\dagger}\left|U_{\dagger}(y)\right|^{2}\right) d x>0
$$

ensures that $\underline{\sigma}^{\varepsilon}<\Lambda_{\dagger}$, meaning that the discrete spectrum of $A^{\varepsilon}$ is nonempty and that $\lambda_{1}=\underline{\sigma}^{\varepsilon}$ is the smallest eigenvalue.

If $I_{\dagger}\left(U_{\dagger}\right)<0$, then the variational method says nothing about the discrete spectrum, but if

$$
I_{\dagger}\left(U_{\dagger}\right)=0,
$$

which is typical of curved screens of zero thickness, then we can still get information. As in [9], we modify the test function:

$$
u_{v}^{\varepsilon \delta}(x)=\exp (-\delta|z|) U_{\dagger}(y)+\sqrt{\delta} v(x) ;
$$

here $v$ is an arbitrary smooth function with small support $\operatorname{supp} v$ in a neighborhood of the screen $\Theta^{\varepsilon}$. Using (1.24) and (1.21), we reshape inequality (1.20) (which is true by assumption) as follows:

$$
2 \sqrt{\delta} \Lambda_{\dagger}\left(U_{\dagger}, v\right)_{\Omega^{\varepsilon}}-c_{v} \delta \leq 2 \sqrt{\delta}\left(\nabla_{y} U_{\dagger}, \nabla_{y} v\right)_{\Omega^{\varepsilon}}+c_{v} \delta .
$$

Note that integration is over the compact set $\operatorname{supp} v$, i.e., all integrals converge.

Now, recalling that $U_{\dagger}=\left.u^{\varepsilon \delta}\right|_{\delta=0} \in \stackrel{\circ}{H}^{1}(\omega ; \partial \omega)$ solves problem (1.10), and applying the Green formula, we replace (1.25) with the inequality

$$
\sqrt{\delta}\left(\partial_{\nu} U_{\dagger}, v\right)_{\partial \Theta^{\varepsilon}} \leq c_{v} \delta
$$

which can easily be made false for small $\delta>0$ by choosing an appropriate function $v$, provided that the normal derivative of the "stationary" wave (1.17) does not vanish everywhere on $\theta_{ \pm}^{\varepsilon}$, i.e., provided that

$$
\partial_{\nu} U_{\dagger} \not \equiv 0 \quad \text { on } \quad \theta_{+}^{\varepsilon} \cup \theta_{-}^{\varepsilon} .
$$

The identity $\partial_{\nu} U_{\dagger}=0$ a.e. on $\partial \Theta^{\varepsilon}$ is only possible if $\Theta^{\varepsilon}$ is a straight screen of zero thickness orthogonal to the $z$-axis or a union of such screens (cf. Subsection 1 in $\S 4$ ), i.e., if we rectify completely the screens in Figures $2 \mathrm{~b}$ and $3 \mathrm{~b}$.

The asymptotic formulas of $\S 2$ will be checked against the two sufficient conditions (1.23) and (1.24), (1.26) for the discrete spectrum to be nonempty, obtained by the variational method. 
3. Remarks on the asymptotic formulas for eigenvalues. The deduction of specific asymptotic formulas for the eigenvalues of a boundary value problem in a regularly or singularly perturbed domain depends heavily on the possibility of finding the exact solution of the arising limit problem. If we perturb a bounded domain, then the spectrum of the limit problem is discrete, the corresponding eigenvalues and eigenfunctions can be thought of as known, and we can try to find the asymptotic corrections via one or other method.

When we are dealing with boundary value problems in unbounded domains, the situation is different, because, as a rule, they have continuous spectrum, so that the limit problem may admit no exact solutions. Nevertheless, for some simple geometric shapes, we can find explicit solutions, which, though, are not eigenfunctions, because they do not decay properly at infinity. As two typical examples, we mention the cylinder $\Omega$ and the half-cylinder $\Omega^{+}=\{x \in \Omega: z>0\}$. In the first case the exact solutions have the form (1.17) and (1.18), while in the second they have the form (1.17) or (1.18) depending on what boundary conditions, Dirichlet or Neumann, are posed on the "end-wall" of the half-cylinder. These exact solutions were used repeatedly for finding asymptotic formulas for eigenvalues and trapped modes in the case of a regular or singular perturbation of the cylinder (see, e.g., [10, 11, 12, 13), in the case where semistrips are connected by "opened windows" (see [14, 15, 16]), and also in singularly or regularly perturbed problems that describe the fall of a plane wave 1 to a periodic surface or a chain of barriers (see, e.g., [17, 18, 19]).

The observation (discussed in Subsection 2 of $\S 1$ ) about the existence of two solutions of the limit problem in $\Omega \backslash \Theta^{0}$ (see formula (1.17) and Lemma 2.1 below) made it possible, in the present paper, to construct the asymptotic expressions for an eigenvalue and an eigenfunction generated by a thin screening barrier $\Theta^{\varepsilon}$ in a cylindrical waveguide. It should be emphasized that no formula is available for a solution that grows linearly at infinity, but the construction of asymptotic expressions does not require such an explicit formula.

The difference (already mentioned) between asymptotic representations of eigenvalues in bounded and unbounded domains can be illustrated by the example of the following mixed boundary value problem in the finite cylinder $\Omega_{L}=\omega \times(-L, L)$ with a cavity of the form (1.1):

$$
\begin{aligned}
-\Delta_{x} v^{\varepsilon}(x) & =\kappa^{\varepsilon} v^{\varepsilon}(x), \quad x \in \Omega_{L}^{\varepsilon}=\Omega_{L} \backslash \Theta^{\varepsilon}, \\
v^{\varepsilon}(x)=0, \quad x \in \Gamma_{L} & =\partial \omega \times(-L, L), \quad \partial_{\nu} v^{\varepsilon}(x)=0, \quad x \in \partial \Omega \backslash \overline{\Gamma_{L}} .
\end{aligned}
$$

For clear reasons, the limit $(\varepsilon=0)$ problem (1.27) in the cylinder $\Omega_{L}^{0}=\Omega_{L} \backslash \Theta^{0}$ with cut has an eigenvalue $\kappa_{1}^{0}=\Lambda_{\dagger}$, which gives rise to the eigenfunction

$$
v_{1}^{0}(x)=L^{-1 / 2} U_{\dagger}(y)
$$

normalized in $L_{2}\left(\Omega_{L}^{0}\right)$.

Note that the function (1.28) is positive inside $\Omega_{L}^{0}$; hence, $\kappa_{1}^{0}$ is the first (smallest) eigenvalue, and it is simple (see, e.g., [2]).

By using the calculation in $\S 2$ and the asymptotic procedures presented in [7, Chapter 9 and $§ 5.5]$, it is not hard to verify the following claims.

Proposition 1.1. The first eigenvalue $\kappa_{1}^{\varepsilon}$ of problem (1.27) admits the expansion

$$
\kappa_{1}^{\varepsilon}=\Lambda_{\dagger}-\varepsilon L^{-1} \int_{\theta} H(y)\left(\left|\nabla_{y} U_{\dagger}(y)\right|^{2}-\Lambda_{\dagger}\left|U_{\dagger}(y)\right|^{2}\right) d y+\widetilde{\kappa}_{1}^{\varepsilon} .
$$

\footnotetext{
${ }^{1}$ When quasiperiodicity conditions are posed (see, e.g., [4]), the Helmholtz equation in a half-plane reduces to the same equation in a half-strip.
} 
If the integral occurring here vanishes, then this expansion can be specified as follows:

$$
\kappa_{1}^{\varepsilon}=\Lambda_{\dagger}-\varepsilon^{2} L^{-1} \int_{\Omega_{L}^{0}}\left(\left|\nabla_{x} v^{\prime}(x)\right|^{2}-\Lambda_{\dagger}\left|v^{\prime}(x)\right|^{2}\right) d x+\widetilde{\kappa}_{1}^{\varepsilon},
$$

where $v^{\prime}$ is a solution of the problem

$$
\begin{aligned}
-\Delta_{x} v^{\prime}(x) & =\Lambda_{\dagger} v^{\prime}(x), \quad x \in \Omega_{L}^{0}, \quad v^{\prime}(x)=0, \quad x \in \Gamma_{L}, \\
\pm \partial_{z} v^{\prime}(y, \pm L) & =0, \quad y \in \omega, \quad \mp \partial_{z} v^{\prime}(y, \pm 0)=-\nabla_{y} H_{ \pm}(y) \cdot \nabla_{y} U_{\dagger}(y), \quad y \in \theta .
\end{aligned}
$$

The remainder terms in (1.29) and (1.30) satisfy, for instance, the estimates $\left|\widetilde{\kappa}_{1}^{\varepsilon}\right| \leq c \varepsilon^{3 / 2}$ and $\left|\widetilde{\widetilde{\kappa}}_{1}^{\varepsilon}\right| \leq c \varepsilon^{5 / 2}$.

Remark 1.1. The quantity $\Lambda_{\dagger}$ serves as the first eigenvalue also for the mixed boundary-value problem in the entire cylinder $\Omega_{L}$ (without cut). By the minimum principle (see, e.g., [3, Theorem 10.2.1]), we have

$$
\kappa_{1}^{\varepsilon}=\inf _{v \in \dot{H}^{1}\left(\Omega_{L}^{\varepsilon} ; \Gamma_{L}\right)} \frac{\left\|\nabla_{x} v ; L_{2}\left(\Omega_{L}^{\varepsilon}\right)\right\|^{2}}{\left\|v ; L_{2}\left(\Omega_{L}^{\varepsilon}\right)\right\|^{2}} .
$$

In the case of a screen of zero thickness, the space $\stackrel{\circ}{H}^{1}\left(\Omega_{L}^{\varepsilon} ; \Gamma_{L}\right)$ is wider than $\stackrel{\circ}{H}^{1}\left(\Omega_{L} ; \Gamma_{L}\right)$ (jumps of functions on $\Theta^{\varepsilon}$ are allowed), so that $\kappa_{1}^{\varepsilon}<\Lambda_{\dagger}$ by the minimum principle. This agrees with (1.30), because in Subsection 4 of $\S 2$ (see (2.39) ) it will be shown that the integral in (1.30) is positive in this case.

In distinction to formulas (1.29) and (1.30), the asymptotic corrections for the eigenvalues of problem (1.5) - (1.7) in an infinite waveguide are of orders of $\varepsilon^{2}$ and $\varepsilon^{4}$, respectively (see Theorems 3.1 and 3.2), and cannot be obtained by the limit passage as $L \rightarrow+\infty$. The ways of obtaining such corrections also differ. For a bounded domain, the correction formulas are deduced from the solvability conditions that serve problems for the lower order terms (see [6, 7]), but in the case where the waveguide in question is unbounded, they are based on the natural condition of the trapped mode decay (see Subsection 3 of $\S 2)$.

The justification of asymptotic formulas also requires a new approach, because the usual tool, the lemma about "near" eigenvalues and eigenfunctions [20, assumes that the problem operator is compact. By the present time, several approaches are developed to justification of asymptotic formulas (see, e.g., the papers [12]-18] cited above); they invoke the Green function poles, the extended scattering matrix, and other tools. In this paper, the author prefers to employ the spectral theory techniques (see 3. Chapter 5 and 6]), because they are easy to use, do not require the compactness of the operator, and allow us to apply the same standard computations as when employing the classical lemma about "near eigenvalues."

\section{§2. Formal asymptotic Constructions}

1. Two solutions of the homogeneous limit problems at the threshold of the continuous spectrum. We want to make use of the results of the theory of elliptic boundary value problems in domains with cylinder-like outlets to infinity (see the key papers [21, 22, 23, 24] and also, e.g., the books [25, 26]). For this, we introduce the Sobolev space $W_{\beta}^{l}\left(\Omega^{0}\right)$ with exponential weight as the completion of the linear set2

\footnotetext{
${ }^{2}$ The functions that are infinitely differentiable up to the boundary and have compact support in $\overline{\Omega^{0}}$; jumps on the shores of cuts are allowed.
} 


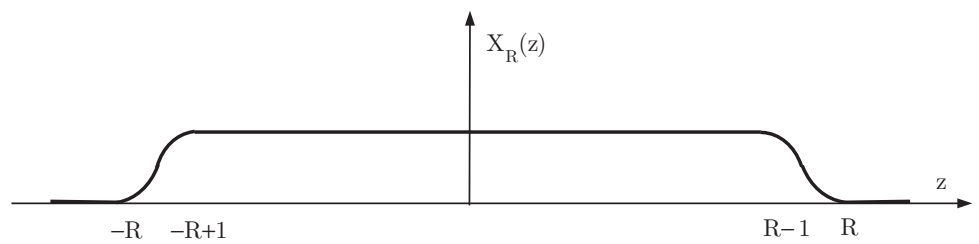

Figure 4. The graph of the cutoff function.

$C_{c}^{\infty}\left(\Omega^{0}\right)$ with respect to the norm

$$
\left\|w ; W_{\beta}^{l}\left(\Omega^{0}\right)\right\|=\left(\sum_{k=0}^{l}\left\|\exp (\beta|z|) \nabla_{x}^{k} w ; L_{2}\left(\Omega^{0}\right)\right\|^{2}\right)^{1 / 2} .
$$

Here $\beta \in \mathbb{R}$ and $l \in \mathbb{N}_{0}=\{0,1,2, \ldots\}$ are the weight and smoothness exponents, and $\nabla_{x}^{k} w$ is the collection of derivatives of $w$ of order $k$. The space $W_{\beta}^{l}\left(\Omega^{0}\right)$ consists of all functions $w \in H_{l o c}^{l}\left(\Omega^{0}\right)$ for which the norm (2.1) is finite. We denote by $\stackrel{\circ}{W}_{\beta}^{1}\left(\Omega^{0} ; \Gamma\right)$ the subspace of functions that vanish on $\Gamma=\partial \Omega$.

Having fixed the parameter $\lambda$, we consider the nonhomogeneous problem (1.13), (1.14) stated as the integral identity

$$
\left(\nabla_{x} w, \nabla_{x} v\right)_{\Omega^{0}}-\lambda(w, v)_{\Omega^{0}}=f(v), \quad v \in \stackrel{\circ}{W}_{-\beta}^{1}\left(\Omega^{0} ; \Gamma\right),
$$

where $(,)_{\Omega^{0}}$ is the extension of the scalar product in $L_{2}\left(\Omega^{0}\right)$ up to duality between the weighted Lebesgue spaces $W_{\beta}^{0}\left(\Omega^{0}\right)$ and $W_{-\beta}^{0}\left(\Omega^{0}\right)$ (note that $W_{\beta}^{l}\left(\Omega^{0}\right) \subset W_{\beta}^{0}\left(\Omega^{0}\right)$ by (2.1) $)$. In problem (2.2), given a linear functional $f \in \stackrel{\circ}{W}_{-\beta}^{1}\left(\Omega^{0}\right)^{*}$, we seek a function $\stackrel{\circ}{W}_{-\beta}^{1}\left(\Omega^{0}\right)$ that satisfies (2.2) for any test function $w \in \stackrel{\circ}{W}_{\beta}^{1}\left(\Omega^{0}\right)$. If $\beta=0$, then $W_{ \pm \beta}^{1}\left(\Omega^{0}\right)=H^{1}\left(\Omega^{0}\right)$, and (2.2) becomes a usual variational problem. Then inequality (1.16) guarantees the unique solvability of this problem for $\lambda<\Lambda_{\dagger}$. However, for $\lambda \geq \Lambda_{\dagger}$ this property may fail; moreover, it is known (see [21] and [25, Theorem 3.1.1]) that the operator of problem (2.2), viewed as a man 3

$$
\mathcal{A}_{\beta}^{0}: \stackrel{\circ}{W}_{\beta}^{1}\left(\Omega^{0}\right) \rightarrow \stackrel{\circ}{W}_{-\beta}^{1}\left(\Omega^{0}\right)^{*},
$$

even ceases to be Fredholm for $\beta=0$. In spectral theory, this fact means precisely that $\lambda$ belongs to the continuous spectrum of the operator $A^{0}$ : the Weyl singular sequence (see [3, §9.1]) can be constructed along the standard lines. For instance, for $\lambda=\Lambda_{\dagger}$, the elements of such a sequence have the form $N^{-1 / 2} X_{2^{N}}\left(z-2^{N+1}\right) U_{\dagger}(y)$, where $N \in \mathbb{N}=$ $\{1,2, \ldots\}, w^{0}$ is the "stationary" wave (1.17), and $X_{R}$ is a cutoff function with graph as in Figure 4 (the details can be found, e.g., in [27]).

The advantage of the general theory that we are going to apply is in the possibility to handle any weight indices $\beta$ in formula (2.3) . The operator $\mathcal{A}_{\beta}^{0}$ turns out to be Fredholm for all $\beta \in \mathbb{R}$ except for a countable collection of forbidden indices (among which we find $\beta=0$ for $\lambda \geq \Lambda_{\dagger}$ ). In particular, $\mathcal{A}_{\beta}^{0}$ is a Fredholm monomorphism for $\beta \in\left(0, \beta^{0}\right)$ with a small $\beta^{0}>0$. We check that the kernel of $\mathcal{A}_{\beta}^{0}$ is trivial (as to the Fredholm property itself, $\mathcal{A}_{\beta}^{0}$ is Fredholm only if $\beta \neq \pm \operatorname{Re} \sqrt{\Lambda_{p}-\lambda}$, where $p \in \mathbb{N}$ and $\Lambda_{1}, \Lambda_{2}, \ldots$ are the eigenvalues of the Dirichlet problem (1.10) on the cross section $\omega$; see [25, Chapters 2 and 4]).

\footnotetext{
${ }^{3}$ As a rule, the theory of elliptic boundary value problems with cylindrical outlets to infinity deals with classically posed problems. However, the passage to weak solutions meets no serious difficulties; see 27 .
} 
The homogeneous $(f=0)$ problem (2.2) with $\lambda=\Lambda_{\dagger}$ cannot have a nontrivial solution $w \in \stackrel{\circ}{W}_{\beta}^{1}\left(\Omega^{0}\right)$, i.e., $\mathcal{A}_{\beta}^{0}$ is a monomorphism for every $\beta>0$. Indeed, by (1.16), for such a solution we have

$$
-\left\|\partial_{z} w ; L_{2}\left(\Omega^{0}\right)\right\|^{2}=\left\|\nabla_{y} w ; L_{2}\left(\Omega^{0}\right)\right\|^{2}-\Lambda_{\dagger}\left\|w ; L_{2}\left(\Omega^{0}\right)\right\|^{2} \geq 0,
$$

whence $\partial_{z} w=0$, and $w=0$ because of decay at infinity.

Since $\mathcal{A}_{-\beta}^{0}$ is the adjoint to $\mathcal{A}_{\beta}^{0}$, the operator $\mathcal{A}_{\gamma}^{0}$ is an epimorphism for any $\gamma<0$. In other words, if $\beta>0$ and $f \in \stackrel{\circ}{W}_{-\beta}^{1}\left(\Omega^{0}\right)^{*}$, then problem (2.2) admits at most one solution $w \in \stackrel{\circ}{W}_{\beta}^{1}\left(\Omega^{0}\right)$ decaying exponentially at infinity, and if $\gamma<0$, then for any right-hand side $f \in \stackrel{\circ}{W}_{-\gamma}^{1}\left(\Omega^{0}\right)^{*}$ problem (2.2) has a solution $w \in \stackrel{\circ}{W}_{\gamma}^{1}\left(\Omega^{0}\right)$ that may have a certain exponential growth at infinity.

Now we apply the theorem on the index increment (see [25, Theorem 4.3.3]), which shows that, in the case under consideration, for all small (hence, not forbidden) indices $\beta>0$ we have

$$
\text { Ind } \mathcal{A}_{-\beta}^{0}=\operatorname{Ind} \mathcal{A}_{\beta}^{0}+N \varkappa=\operatorname{Ind} \mathcal{A}_{\beta}^{0}+4,
$$

where $N=2$ is the number of exits to infinity $(z \rightarrow \pm \infty)$ for the domain $\Omega^{0}$, and $\varkappa=2$ is the dimension of the subspace of the polynomial (in $z$ ) solutions of the homogeneous Dirichlet problem in the cylinder $\Omega=\omega \times \mathbb{R}$ for the Helmholtz operator $-\Delta_{x}-\Lambda_{\dagger}$ (it is easily seen that (1.17) and (1.18) are the only "polynomial" solutions). We have

$$
\text { Ind } \mathcal{A}_{\beta}^{0}=\operatorname{dim} \operatorname{ker} \mathcal{A}_{\beta}^{0}-\operatorname{dim} \text { coker } \mathcal{A}_{\beta}^{0} \text {, }
$$

where ker $\mathcal{A}_{\beta}^{0}$ and coker $\mathcal{A}_{\beta}^{0}=\operatorname{ker} \mathcal{A}_{-\beta}^{0}$ are the kernel and the cokernel of $\mathcal{A}_{\beta}^{0}$ and, by the said above,

$$
\text { Ind } \mathcal{A}_{\beta}^{0}=-\operatorname{Ind} \mathcal{A}_{-\beta}^{0}, \quad \operatorname{dim} \operatorname{ker} \mathcal{A}_{\beta}^{0}=\operatorname{dim} \operatorname{coker} \mathcal{A}_{-\beta}^{0}=0 .
$$

Summarizing, we see that

$$
\operatorname{dim} \operatorname{ker} \mathcal{A}_{-\beta}^{0}=\operatorname{Ind} \mathcal{A}_{-\beta}^{0}=2 \text {. }
$$

Clearly, $w^{0}=U_{\dagger} \in \stackrel{\circ}{W}_{-\beta}^{1}\left(\Omega^{0}\right)$, but (2.4) implies that the homogeneous problem (2.2) has yet another solution $w^{\prime}$ linearly independent of $w^{0}$ and belonging to the space $\stackrel{\circ}{W}_{\gamma}^{1}\left(\Omega^{0}\right)$ for every negative weight index $\gamma$. Using the theorem on asymptotic behavior from [21] (see [25, Theorem 3.1.4]) and recalling the said above about polynomial solutions of the Helmholtz equation in the cylinder $\omega \times \mathbb{R}$, we deduce the representation

$$
w^{\prime}(x)=\sum_{ \pm}(1-\chi( \pm z))\left(c_{0}^{ \pm}+c_{1}^{ \pm} z\right) U_{\dagger}(y)+\widetilde{w}^{\prime}(x),
$$

where $\chi \in C^{\infty}(\mathbb{R})$ is a cutoff function, $\chi(z)=0$ for $z>1$, $\chi(z)=1$ for $z<0$, the $c_{q}^{ \pm}$are some coefficients, and $\widetilde{w}^{\prime} \in \stackrel{\circ}{W}_{\beta}^{1}\left(\Omega^{0}\right)$ is an exponentially decaying remainder. To determine the coefficients $c_{1}^{ \pm}$, we apply the method of [22] (see also [25, §3.2]), substituting $w^{0}$ and $w^{\prime}$ in the Green formula on the set $\Omega_{R}^{0}=\left\{x \in \Omega^{0}:|z|<R\right\}$, which is a long, but finite cylinder with screen:

$$
\begin{aligned}
0 & =\int_{\Omega_{R}^{0}}\left(w^{\prime}(x)\left(\Delta_{x} w^{0}(x)+\Lambda_{\dagger} w^{0}(x)\right)-w^{0}(x)\left(\Delta_{x} w^{\prime}(x)+\Lambda_{\dagger} w^{\prime}(x)\right)\right) d x \\
& =\int_{\omega}\left(w^{\prime}(y, R) \partial_{z} w^{0}(y, R)-w^{0}(y, R) \partial_{z} w^{\prime}(y, R)\right) d y \\
& =\sum_{ \pm} \mp c_{1}^{ \pm} \int_{\omega}\left|U_{\dagger}(y)\right|^{2} d y+O(\exp (-R \beta))=c_{1}^{-}-c_{1}^{+}+O(\exp (-R \beta))
\end{aligned}
$$


Passing to the limit as $R \rightarrow+\infty$, we see that $c_{1}^{-}=c_{1}^{+}$. Now we show that the relation $c_{1}^{ \pm}=0$ is impossible. Putting

$$
w^{\prime}(y, z)=W^{\prime}(z) U_{\dagger}(y)+w^{\perp}(y, z), \quad W^{\prime}(z)=\int_{\omega} w^{\prime}(y, z) U_{\dagger}(y) d y,
$$

we multiply $w^{\prime}$ by the cutoff function

$$
\chi_{R}(z)=\chi(R+z) \chi(R-z), \quad 0 \leq \chi_{R} \leq 1
$$

(see its graph in Figure 5); here $\chi$ is the same cutoff function as in (2.5). We take the result $v=\chi_{R} w^{0} \in \stackrel{\circ}{H}^{1}\left(\Omega^{0} ; \Gamma\right)$ for the role of a test function in (2.2) with $f=0$. Since formulas (1.11) and (2.7) show that

$$
\int_{\omega} w^{\perp}(y, z) U_{\dagger}(y) d y=0 \quad \text { for a.e. } \quad z \in \mathbb{R}
$$

and moreover,

$$
\left(\chi_{R} W^{\prime} \nabla_{y} U_{\dagger}, \nabla_{y} w^{\prime}\right)_{\Omega^{0}}-\Lambda_{\dagger}\left(\chi_{R} W^{\prime} U_{\dagger}, w^{\prime}\right)_{\Omega^{0}}=-\left(\chi_{R} W^{\prime}\left(\Delta_{y} U_{\dagger}+\Lambda_{\dagger} U_{\dagger}\right), w^{\prime}\right)_{\Omega^{0}}=0,
$$

we have

$$
\begin{aligned}
0= & \left(\chi_{R} \nabla_{x} w^{\prime}, \nabla_{x} w^{\prime}\right)_{\Omega^{0}}-\Lambda_{\dagger}\left(\chi_{R} w^{\prime}, w^{\prime}\right)_{\Omega^{0}}+\left(\nabla_{x} w^{\prime}, w^{\prime} \nabla_{x} \chi_{R}\right)_{\Omega^{0}} \\
= & \int_{\Omega^{0}} \chi_{R}(z)\left|\partial_{z} W^{\prime}(z)\right|^{2}\left|U_{\dagger}(y)\right|^{2} d x+\int_{\Omega^{0}} \chi_{R}(z)\left|\nabla_{x} w^{\perp}(x)\right|^{2} d x \\
& \quad-\Lambda_{\dagger} \int_{\Omega^{0}} \chi_{R}(z)\left|w^{\perp}(x)\right|^{2} d x+\int_{\Omega^{0}} w^{\prime}(x) \partial_{z} \chi_{R}(z) \partial_{z} w^{\prime}(x) d x .
\end{aligned}
$$

Two facts should be taken into account. First, since it has been assumed that $c_{1}^{ \pm}=0$, formula (2.5) shows that the derivative $\partial_{z} w^{\prime}(y, z)$ decays exponentially as $z \rightarrow \pm \infty$, and $\partial_{z} \chi_{R}(z) \neq 0$ only if $|z| \in[R-1, R]$. Therefore,

$$
\left|\int_{\Omega^{0}} w^{\prime}(x) \partial_{z} \chi_{R}(z) \partial_{z} w^{\prime}(x) d x\right| \leq c \exp (-\beta R)
$$

Second, for the function $w^{\perp}$, which satisfies the orthogonality condition (2.9) on the cross section $\omega$, the Poincaré inequality is true:

$$
\int_{\omega}\left|\nabla_{y} w^{\perp}(y, z)\right|^{2} d y \geq \Lambda_{2} \int_{\omega}\left|w^{\perp}(y, z)\right|^{2} d y \quad \text { for a.e. } \quad z \in \mathbb{R},
$$

where $\Lambda_{2}>\Lambda_{1}=\Lambda_{\dagger}$ is the second eigenvalue of problem (1.10). We multiply (2.11) by $\chi_{R}(z)$ and integrate over $z \in \mathbb{R}$ (the cut $\Theta^{0}$ occurring in $\Omega$ does not impede this), obtaining

$$
\int_{\Omega^{0}} \chi_{R}(z)\left|\nabla_{x} w^{\perp}(y, z)\right|^{2} d x \geq \Lambda_{2} \int_{\Omega^{0}} \chi_{R}(z)\left|w^{\perp}(y, z)\right|^{2} d x .
$$

So, formula (2.10) implies the relation

$$
0 \geq\left(\Lambda_{2}-\Lambda_{1}\right)\left\|\chi_{R}^{1 / 2} w^{\perp} ; L_{2}\left(\Omega^{0}\right)\right\|^{2}+C \exp (-\beta R) \quad \text { as } \quad R \rightarrow+\infty,
$$

whence $w^{\perp}=0$. It remains to observe that the function $w^{\prime}(y, z)=W(z) U_{\dagger}(y)$ can solve problem (1.13) with $\lambda=\Lambda_{\dagger}$ only if $W(z)=$ const, but since the solution $w^{1}$ is linearly independent of $w^{0}$, it cannot be proportional to $w^{0}=U_{\dagger}$, so that the supposition $c_{1}^{ \pm}=0$ is false. To normalize the second solution, we put

$$
w^{1}(x)=\left(c_{1}^{ \pm}\right)^{-1}\left(w^{\prime}(x)-\frac{1}{2}\left(c_{0}^{+}+c_{0}^{-}\right)\right) .
$$

Lemma 2.1. For small $\beta>0$, every solution $w \in \stackrel{\circ}{W}_{-\beta}^{1}\left(\Omega^{0}\right)$ of the homogeneous $(f=0)$ problem (2.2) is a linear combination of the solutions (1.17) and (2.13). 
Remark 2.1. The boundary of the domain $\Omega^{0}$ has irregularities only on the edge $\vartheta^{0}=$ $\{(y, z): y \in \partial \theta, z=0\}$ of the screen $\Theta^{0}$, i.e., the solution $w^{1}$ is smooth everywhere except a neighborhood $\mathcal{V}$ of this edge. The weighted Hölder estimates (see [23] and also [25. §3.6]) for solutions of elliptic boundary value problems in cylindrical domains show that the asymptotic representation (2.5) for the special solution (2.13) can be rewritten as follows:

$$
\left|\nabla_{x}^{k}\left(w^{1}(x)-z U_{\dagger}(y) \mp b\right)\right| \leq c_{k}(d) \exp \left(\mp z \sqrt{\Lambda_{2}-\Lambda_{1}}\right) \text { for } \quad \pm z>d .
$$

Here $k \in \mathbb{N}_{0}=\{0,1,2, \ldots\}, d$ is an arbitrary positive number, and $b=\frac{1}{2}\left(c_{1}^{ \pm}\right)^{-1}\left(c_{0}^{+}-c_{0}^{-}\right)$ is a constant depending on the shape of the screen $\theta$ and the cross section $\omega$.

2. The main correction term in asymptotic formulas for eigenfunction. The set $\Theta^{\varepsilon}$ should be viewed as a regular perturbation of the $(n-1)$-dimensional screen $\Theta^{0}$ (see [7, §5.5]); therefore, it is reasonable to adopt the following asymptotic Ansatz:

$$
u^{\varepsilon}(x)=w^{0}(x)+\varepsilon w^{\prime}(x)+\cdots .
$$

The role of the leading term of the asymptotic expansion for a possible eigenfunction $u^{\varepsilon} \in \stackrel{\circ}{H}^{1}\left(\Omega^{\varepsilon} ; \Gamma\right)$ is played by the solution (1.17) of the limit problem (1.12), (1.14) with the "best" behavior at infinity (i.e., the bounded solution; note that the solution $w^{1}$ has linear growth). Nevertheless, the function $w^{1}$ will be employed in the next section for constructing the final form of the correction term $w^{\prime}$. It should be emphasized that, in any case, the asymptotic Ansatz (2.15) will need substantial correction, because the eigenfunction (trapped mode) must belong to the Sobolev space, and hence, must decay exponentially at infinity.

The unit vector $\nu_{ \pm}^{\varepsilon}(y)$ normal to the surfaces of the screen (1.4), outward relative to the domain $\Omega^{\varepsilon}=\Omega \backslash \Theta^{\varepsilon}$, looks like this:

$$
\nu_{ \pm}^{\varepsilon}(y)=\left[1+\varepsilon^{2}\left|\nabla_{y} H_{ \pm}(y)\right|^{2}\right]^{-1 / 2}\left(\varepsilon \nabla_{y} H_{ \pm}(y), \mp 1\right) .
$$

Hence, the operator of differentiation along the normal satisfies the relation

$$
\left[1+\varepsilon^{2}\left|\nabla_{y} H_{ \pm}(y)\right|^{2}\right]^{1 / 2} \partial_{\nu}=\mp \partial_{z}+\varepsilon \nabla_{y} H_{ \pm}(y) \cdot \nabla_{y} .
$$

Here and in the sequel, the dot means the scalar product in $\mathbb{R}^{n-1}$.

We substitute (2.15) in the boundary condition (1.7) on the surfaces (1.4). Besides the fact that the differential operator (2.17) depends on the parameter $\varepsilon$, we must take into account the deviation of the surface $\theta_{ \pm}^{\varepsilon}$ from the limit position $\theta_{ \pm}^{0}=\theta \times\{ \pm 0\}$; hence, we must carry the data over to the rectified screen (this is easy at the first step of the algorithm, but in the sequel, in $\S 4$, we shall need the Taylor formula). Thus,

$$
\begin{aligned}
& {\left[1+\varepsilon^{2} \mid \nabla_{y} H_{ \pm}(y) \|^{2}\right]^{1 / 2} \partial_{\nu} u^{\varepsilon}\left(y, \pm \varepsilon H_{ \pm}(y)\right)} \\
& \quad=\varepsilon\left(\mp \partial_{z} w^{\prime}(y, \pm 0)+\nabla_{y} H_{ \pm}(y) \cdot \nabla_{y} U_{\dagger}(y)\right)+\cdots .
\end{aligned}
$$

Equating the leading term of the discrepancy (2.18) to zero, we make the correction $w^{\prime}$ subject to the following boundary conditions:

$$
\mp \partial_{z} w^{\prime}(y, \pm 0)=-\nabla_{y} H_{ \pm}(y) \cdot \nabla_{y} U_{\dagger}(y), \quad y \in \theta .
$$

Assuming that $\lambda^{\varepsilon}=\Lambda_{\dagger}+o(\varepsilon)$ (this assumption will be justified formally in the next section and completely in $\S 3$ ), we show that the differential equation (1.5) and the boundary condition (1.6) imply the relations

$$
-\Delta_{x} w^{\prime}(x)=\Lambda_{\dagger} w^{\prime}(x), x \in \Omega^{0}, \quad w^{\prime}(x)=0, x \in \Gamma .
$$

In accordance with what was said in the preceding section about the operator of problem (2.2) and the asymptotic representations of its solutions, problem (2.20), (2.19) (the statement of which as an integral identity is obvious) has a solution (2.5) of linear 
growth at infinity, which is determined up to a linear combination $C_{0}^{\prime} w^{0}+C_{1}^{\prime} w^{1}$. Using (1.17) and (2.14), we see that the coefficients of this linear combination can be chosen in such a way that

$$
\left|\nabla_{x}^{k}\left(w^{\prime}(x) \mp\left(b_{0}^{\prime}+b_{1}^{\prime} z\right) U_{\dagger}(y)\right)\right| \leq c_{d k} \exp \left(\mp z \sqrt{\Lambda_{2}-\Lambda_{1}}\right) \text { for } \pm z>d,
$$

where $d>0$ is an arbitrary number (see Remark 2.1). Indeed, in the formula $w^{\prime}=$ $\mathbf{w}^{\prime}+C_{1}^{\prime} w^{1}+C_{0}^{\prime} w^{0}$, where $\mathbf{w}^{\prime}$ is a particular solution, we can choose $C_{1}^{\prime}$ so that the factors become equal as $\pm z \rightarrow+\infty$, and then $C_{0}^{\prime}$ is chosen so that $w^{\prime}(y, z) \mp b_{1}^{\prime} z \rightarrow \pm b_{0}^{\prime}$ as $\pm z \rightarrow+\infty$.

The coefficient $b_{1}^{\prime}$ that has emerged will be computed with the help of the method of 22] (cf. the calculations (2.6)):

$$
\begin{aligned}
2 b^{\prime} & =\lim _{R \rightarrow \infty} \sum_{ \pm} \pm \int_{\omega} U_{\dagger}(y) \partial_{z} w^{\prime}(y, \pm R) d y \\
& =\lim _{R \rightarrow \infty}\left(\int_{\Omega_{R}^{0}} U_{\dagger}(y)\left(\Delta_{x} w^{\prime}(x)+\Lambda_{\dagger} w^{\prime}(x)\right) d x+\sum_{ \pm} \pm \int_{\theta} U_{\dagger}(y) \partial_{z} w^{\prime}(y, \pm 0) d y\right) \\
& =\sum_{ \pm} \pm \int_{\theta} U_{\dagger}(y) \nabla_{y} H_{ \pm}(y) \cdot \nabla_{y} U_{\dagger}(y) d y=\int_{\theta} U_{\dagger}(y) \nabla_{y} H(y) \cdot \nabla_{y} U_{\dagger}(y) d y \\
& =-\int_{\theta} H(y)\left|\nabla_{y} U_{\dagger}(y)\right|^{2} d y-\int_{\theta} H(y) U_{\dagger}(y) \Delta_{y} U_{\dagger}(y) d y \\
& =-\int_{\theta} H(y)\left(\left|\nabla_{y} U_{\dagger}(y)\right|^{2}-\Lambda_{\dagger}\left|U_{\dagger}(y)\right|^{2}\right) d y=:-J_{\dagger}^{\prime}(H) .
\end{aligned}
$$

Here $H$ is the screen thickness function (1.2), and in the last transformations we have used (1.3) and the Helmholtz equation

$$
-\Delta_{y} U_{\dagger}(y)-\Lambda_{\dagger} U_{\dagger}(y)=0, \quad y \in \omega
$$

for the eigenfunction $U_{\dagger}$ of problem (1.10).

Remark 2.2. The integral in the sufficient condition (1.23), obtained by the variational method [8, 9], is equal to

$$
I_{\dagger}\left(\Theta^{\varepsilon}\right)=\varepsilon \int_{\theta} H(y)\left(\left|\nabla_{y} U_{\dagger}(y)\right|^{2}-\Lambda_{\dagger}\left|U_{\dagger}(y)\right|^{2}\right) d y+O\left(\varepsilon^{2}\right) .
$$

A similar expression $J_{\dagger}^{\prime}(H)$ appeared in (2.22).

In the sequel we distinguish the situations where $J_{\dagger}^{\prime}(H)>0$ and $J_{\dagger}^{\prime}(H)=0$; in either case we shall find eigenvalues on the interval $\left(0, \Lambda_{\dagger}\right)$; the situation with $J_{\dagger}^{\prime}(H)<0$ is discussed in Remarks 2.3 and 3.3 .

3. The main correction term in the asymptotic expansion for an eigenvalue. Suppose that

$$
J_{\dagger}^{\prime}(H)=\int_{\theta} H(y)\left(\left|\nabla_{y} U_{\dagger}(y)\right|^{2}-\Lambda_{\dagger}\left|U_{\dagger}(y)\right|^{2}\right) d y>0 .
$$

Then we adopt the following asymptotic Ansatz for the eigenvalue $\lambda^{\varepsilon} \in\left(0, \Lambda_{\dagger}\right)$ of problem (1.8):

$$
\lambda^{\varepsilon}=\Lambda_{\dagger}-\varepsilon^{2} \mu^{\prime}+\cdots ;
$$


here $\mu^{\prime}$ is a positive quantity to be determined. If $\lambda^{\varepsilon}$ is as in (2.25), then for the solution of problem (1.5) - (1.7) we should take the following expansions as $\pm z \rightarrow+\infty$ :

$$
\begin{aligned}
u^{\varepsilon}(y, z) & =c_{ \pm}^{\varepsilon} U_{\dagger}(y) \exp \left(\mp z \sqrt{\Lambda_{\dagger}-\lambda^{\varepsilon}}\right)+\ldots \\
& =\left(c_{ \pm}^{0}+\varepsilon c_{ \pm}^{\prime}+\cdots\right) U_{\dagger}(y)\left(1 \mp z \sqrt{\varepsilon^{2} \mu^{\prime}+\cdots}\right)+\cdots \\
& =c_{ \pm}^{0} U_{\dagger}(y)+\varepsilon\left(c_{ \pm}^{\prime} \mp c_{\mp}^{0} z \sqrt{\mu^{\prime}}\right)+\cdots
\end{aligned}
$$

The dots hide the lower (as $z \rightarrow \pm \infty$, and also as $\varepsilon \rightarrow+0$ ) asymptotic terms immaterial for our asymptotic analysis. By (1.17) and (2.21), we can write

$$
\begin{array}{r}
w^{0}(x)+\varepsilon w^{\prime}(x)=U_{\dagger}(y)\left(1 \pm \varepsilon\left(b_{1}^{\prime} z+b_{0}^{\prime}\right)+O\left(\exp \left(-|z| \sqrt{\Lambda_{2}-\Lambda_{1}}\right)\right)\right) \\
z \rightarrow \pm \infty
\end{array}
$$

Consequently, putting

$$
\begin{aligned}
c_{ \pm}^{0} & =1, \quad c_{ \pm}^{\prime}= \pm b_{0}^{\prime}, \\
\sqrt{\mu^{\prime}} & =-b_{1}^{\prime} \quad \Rightarrow \quad \mu^{\prime}=\left(b_{1}^{\prime}\right)^{2}=\frac{1}{4} J_{\dagger}^{\prime}(H)^{2},
\end{aligned}
$$

we make the leading terms in (2.26) and (2.27) identical. Thus, we have found (so far formally) the correction to the representation (2.25) of the eigenvalue $\lambda^{\varepsilon}$.

Remark 2.3. The conclusion (2.29) is possible only if $b_{1}^{\prime}<0$, which is ensured by the calculation (2.22) and the assumption (2.23). If the inequality opposite to (2.24) is fulfilled, and hence $b_{1}^{\prime}>0$, then repetition of calculations gives nothing valuable, because $\sqrt{\mu^{\prime}}$ becomes negative and formula (2.26) turns out to be meaningless.

4. The degenerate case. Suppose that

$$
J_{\dagger}^{\prime}(H)=\int_{\theta} H(y)\left(\left|\nabla_{y} U_{\dagger}(y)\right|^{2}-\Lambda_{\dagger}\left|U_{\dagger}(y)\right|^{2}\right) d y=0 .
$$

Then, formulas (2.21) and (2.22) show that problem (2.20), (2.21) has a bounded solution and, moreover, in (2.25) we have $\mu^{\prime}=0$, so that we cannot ensure (even formally) that $\lambda^{\varepsilon} \in\left(0, \Lambda_{\dagger}\right)$.

We modify the asymptotic formulas (2.15) and (2.25):

$$
\begin{aligned}
u^{\varepsilon}(x) & =w^{0}(x)+\varepsilon w^{\prime}(x)+\varepsilon^{2} w^{\prime \prime}(x)+\cdots, \\
\lambda^{\varepsilon} & =\Lambda_{\dagger}-\varepsilon^{4} \mu^{\prime \prime}+\cdots .
\end{aligned}
$$

Using (2.16) and (2.17), we refine relation (2.18) as follows:

$$
\begin{aligned}
{\left[1+\varepsilon^{2} \mid \nabla_{y} H_{ \pm}(y) \|^{2}\right]^{1 / 2} \partial_{\nu} u^{\varepsilon}\left(y, \pm \varepsilon H_{ \pm}(y)\right) } & \\
=\varepsilon & \left(\mp \partial_{z} w^{\prime}(y, \pm 0)+\nabla_{y} H_{ \pm}(y) \cdot \nabla_{y} U_{\dagger}(y)\right)+\varepsilon^{2}\left(\mp \partial_{z} w^{\prime \prime}(y, \pm 0)\right. \\
& \left.+\nabla_{y} H_{ \pm}(y) \cdot \nabla_{y} w^{\prime}(y, \pm 0)-H_{ \pm}(y) \partial_{z}^{2} w^{\prime}(y, \pm 0)\right)+\cdots
\end{aligned}
$$

We have applied the Taylor formula

$$
\partial_{z} w^{\prime}\left(y, \pm \varepsilon H_{ \pm}(y)\right)=\partial_{z} w^{\prime}(y, \pm 0) \pm \varepsilon H_{ \pm}(y) \partial_{z}^{2} w^{\prime}(y, \pm 0)+\cdots .
$$

The first term on the right in (2.33) was already taken into account in the boundary condition (2.19). The second term (the coefficient of $\varepsilon^{2}$ ) vanishes if the function $w^{\prime \prime}$ solves the problem

$$
\begin{aligned}
-\Delta_{x} w^{\prime \prime}(x) & =\Lambda_{\dagger} w^{\prime \prime}(x), \quad x \in \Omega^{0}, \quad w^{\prime \prime}(x)=0, \quad x \in \Gamma \\
\mp \partial_{z} w^{\prime \prime}(y, \pm 0) & =-\nabla_{y} H_{ \pm}(y) \cdot \nabla_{y} w^{\prime}(y, \pm 0)+H_{ \pm}(y) \partial_{z}^{2} w^{\prime}(y, \pm 0), \quad y \in \theta .
\end{aligned}
$$


Assume (see the end of the section) that the right-hand sides of the boundary conditions (2.35) give rise to a continuous functional belonging to $\stackrel{\circ}{H}^{1}\left(\Omega^{0} ; \Gamma\right)^{*}$,

$$
\stackrel{\circ}{H}^{1}\left(\Omega^{0} ; \Gamma\right) \ni v \mapsto \sum_{ \pm} \mp \int_{\theta} v(y, \pm 0)\left(\nabla_{y} H_{ \pm}(y) \cdot \nabla_{y} w^{\prime}(y, \pm 0)-H_{ \pm}(y) \partial_{z}^{2} w^{\prime}(y, \pm 0)\right) d y
$$

Then, for the same reasons as in the preceding sections, problem (2.34), (2.35) admits a solution with linear growth at infinity, and we have inequalities similar to (2.21):

$$
\left|\nabla_{x}^{k} w^{\prime \prime}(x) \mp\left(b_{0}^{\prime \prime}+b_{1}^{\prime \prime} z\right) U_{\dagger}(y)\right| \leq c_{d k} \exp \left(\mp z \sqrt{\Lambda_{2}-\Lambda_{1}}\right) \text { for } \pm z>d
$$

(cf. the text after (2.21) $)$. Repeating the calculations (2.22), we find

$$
2 b^{\prime \prime}=\sum_{\mp} \int_{\theta} U_{\dagger}(y)\left(\nabla_{y} H_{ \pm}(y) \cdot \nabla_{y} w^{\prime}(y, \pm 0)-H_{ \pm}(y) \partial_{z}^{2} w^{\prime}(y, \pm 0)\right) d y .
$$

We transform the right-hand side:

$$
\begin{aligned}
2 b^{\prime}= & \sum_{ \pm} \int_{\theta} U_{\dagger}(y)\left(\nabla_{y} H_{ \pm}(y) \cdot \nabla_{y} w^{\prime}(y, \pm 0)\right. \\
& \left.\quad+H_{ \pm}(y)\left(\Delta_{y} w^{\prime}(y, \pm 0)+\Lambda_{\dagger} w^{\prime}(y, \pm 0)\right)\right) d y \\
= & \sum_{ \pm} \int_{\theta} w^{\prime}(y, \pm 0)\left(H_{ \pm}(y)\left(\Delta_{y} U_{\dagger}(y)+\Lambda_{\dagger} U_{\dagger}(y)\right)+\nabla_{y} H_{ \pm}(y) \cdot \nabla_{y} U_{\dagger}(y)\right) d y \\
= & \sum_{ \pm} \pm \int_{\theta} w^{\prime}(y, \pm 0) \partial_{z} w^{\prime}(y, \pm 0) d y \\
= & -\int_{\Omega^{0}}\left(w^{\prime}(x) \Delta_{x} w^{\prime}(x)+\left|\nabla_{x} w^{\prime}(x)\right|^{2}\right) d x+\sum_{ \pm} \pm \int_{\omega} w^{\prime}(y, \pm R) \partial_{z} w^{\prime}(y, \pm R) d y \\
= & -\int_{\Omega^{0}}\left(\left|\nabla_{x} w^{\prime}(x)\right|^{2}-\Lambda_{\dagger}\left|w^{\prime}(x)\right|^{2}\right) d x+o(1), \quad R \rightarrow+\infty .
\end{aligned}
$$

As an explanation, we note that in the second line we used the integration by parts formula on the $(n-1)$-dimensional domain $\theta$, and the requirement (1.3) ensured the vanishing of the boundary integrals over $\partial \theta$, while in the third and fourth lines we employed the Helmholtz equation (2.23) and the boundary condition (2.19). In the final lines, we integrated by parts once again, now in the finite cylinder $\omega \times(-R, R)$ with screen $\Theta^{0}$, and invoked the asymptotic formula (2.21) with $b_{1}^{\prime}=0$ and with an exponentially decaying remainder term.

In the last integral in (2.38), we apply the expansion (2.7); by the orthogonality condition (2.9) and inequality (2.12), we have

$$
\begin{aligned}
\int_{\Omega_{R}^{0}} & \left(\left|\nabla_{x} w^{\prime}(x)\right|^{2}-\Lambda_{\dagger}\left|w^{\prime}(x)\right|^{2}\right) d x \\
& =\int_{\Omega_{R}^{0}}\left|\partial_{z} W^{\prime}(z)\right|^{2}\left|U_{\dagger}(y)\right|^{2} d x+\int_{\Omega_{R}^{0}}\left(\left|\nabla_{x} w^{\perp}(x)\right|^{2}-\Lambda_{\dagger}\left|w^{\perp}(x)\right|^{2}\right) d x \\
& \geq \int_{-R}^{R}\left|\partial_{z} W^{\prime}(z)\right|^{2} d z+\left(\Lambda_{2}-\Lambda_{1}\right) \int_{\Omega_{R}^{0}}\left|w^{\perp}(x)\right|^{2} d x .
\end{aligned}
$$

The last sum of integrals can be zero only if $W^{\prime}(z)=c^{\prime}$ and $w^{\perp}=0$, i.e., if $w^{\prime}=c^{\prime} w^{0}$, which is impossible. Thus, passing to the limit as $R \rightarrow+\infty$ (we can do this due to 
formula (2.21) with the coefficient $b_{1}^{\prime}=0$ in the linear term), we see that

$$
\begin{aligned}
b_{1}^{\prime \prime} & =-\frac{1}{2} J_{\dagger}^{\prime \prime}(H), \\
J_{\dagger}^{\prime \prime}(H) & =\int_{\mathbb{R}}\left|\partial_{z} W^{\prime}(z)\right|^{2} d z+\int_{\Omega^{0}}\left(\left|\nabla_{x} w^{\perp}(x)\right|^{2}-\Lambda_{\dagger}\left|w^{\perp}(x)\right|^{2}\right) d x>0 .
\end{aligned}
$$

So, we have established formulas (2.36) and (2.40) completely similar to formulas (2.14) and (2.22). Now we repeat the calculations (2.26) keeping the terms of order of $\varepsilon^{2}$, obtaining

$$
\begin{aligned}
u^{\varepsilon}(y, z) & =c_{ \pm}^{\varepsilon} U_{\dagger}(y) \exp \left(\mp z \sqrt{\Lambda_{\dagger}-\lambda^{\varepsilon}}\right)+\cdots \\
& =\left(c_{ \pm}^{0}+\varepsilon c_{ \pm}^{\prime}+\varepsilon^{2} c_{ \pm}^{\prime \prime}+\cdots\right) U_{\dagger}(y)\left(1 \mp z \sqrt{\varepsilon^{4} \mu^{\prime \prime}+\cdots}\right)+\cdots \\
& =c_{ \pm}^{0} U_{\dagger}(y)+\varepsilon c_{ \pm}^{\prime} U_{\dagger}(y)+\varepsilon^{2}\left(c_{ \pm}^{\prime \prime} \mp c_{\mp}^{0} z \sqrt{\mu^{\prime \prime}}\right)+\cdots
\end{aligned}
$$

Comparing this with the expansion

$$
u^{\varepsilon}(y, z)=w^{0}(x)+\varepsilon w^{\prime}(x)+\varepsilon w^{\prime \prime}(x)+\cdots=U_{\dagger}(y)\left(1 \pm \varepsilon b_{0}^{\prime} \pm \varepsilon^{2}\left(b_{0}^{\prime \prime}+b_{1}^{\prime \prime} z\right)\right)+\cdots,
$$

found on the basis of the earlier Ansatz (2.31) with the help of relations (2.21), (2.22), (2.30), and (2.36), we get

$$
\begin{aligned}
c_{ \pm}^{0} & =1, \quad c_{ \pm}^{\prime}= \pm b_{0}^{\prime}, \quad c_{ \pm}^{\prime \prime}= \pm b_{0}^{\prime \prime}, \\
\sqrt{\mu^{\prime \prime}} & =-b_{1}^{\prime \prime} \Rightarrow \mu^{\prime \prime}=\left(b_{1}^{\prime \prime}\right)^{2}=\frac{1}{4} J_{\dagger}^{\prime \prime}(H)^{2} .
\end{aligned}
$$

Relations (2.42), (2.32), and (2.31), as well as (2.25), (2.29), and (2.15), were deduced at the formal asymptotic analysis level and need justification (see $\S 3$ ). Also, in (2.22) and (2.38) we did not pay due attention to the convergence of the integrals over the $(n-1)$-dimensional surfaces $\theta_{ \pm}^{0}$, while the solution $w^{\prime}$ has singularities on the edge $\vartheta^{0}=\{x: y \in \partial \theta, z=0\}$ of the screen (1.9). This convergence is ensured by the assumption (1.3) and the next claim, which is implied by the results of [28, 29, 30] (see also [25, Chapter 8 and 9]) and extends Remark 2.1]

Lemma 2.2. In a neighborhood $\mathcal{V}$ of the edge $\vartheta^{0}$ of the screen $\Theta^{0}$, the solutions $w^{\prime}$ and $w^{\prime \prime}$ of problems (2.20), (2.19) and (2.34), (2.35) satisfy the estimates

$$
\left|w^{\cdots}(x)\right| \leq c_{0}, \quad\left|\nabla_{x}^{k} w^{\cdots}(x)\right| \leq c_{k \delta} \rho(x)^{-\delta-k+1 / 2}, \quad k \in \mathbb{N},
$$

where $\rho(x)=\operatorname{dist}\left(x, \partial \vartheta^{0}\right)$, and $\delta$ is an arbitrary positive number; generally speaking, for $w^{\prime \prime}$ we have $c_{k \delta} \rightarrow+\infty$ as $\delta \rightarrow+0$.

Estimates (2.43) show that the integrand $U_{\dagger}(y) \partial_{z} w^{\prime}(y, \pm 0)$ in (2.22) has a singularity $O\left(\rho^{-\delta-1 / 2}\right)$, so that all the integrals converge. Moreover, since

$$
\begin{aligned}
& \left|\nabla_{y} H_{ \pm}(y) \cdot \nabla_{y} w^{\prime}(y, \pm 0)\right|+\left|w^{\prime}(y, \pm 0) \partial_{z} w^{\prime}(y, \pm 0)\right| \leq c \rho(y, 0)^{-\delta-1 / 2}, \\
& \left|H_{ \pm}(y) \nabla_{y}^{2} w^{\prime}(y, \pm 0)\right| \leq c \rho(y, 0) \rho(y, 0)^{-\delta-3 / 2}=c \rho(y, 0)^{-\delta-1 / 2},
\end{aligned}
$$

no convergence problems arise also in (2.38).

\section{§3. JUSTIFICATION OF ASYMPTOTIC FORMULAS}

1. The global asymptotic construction. Now we try to justify the formal asymptotic analysis presented in Subsections 2 and 3 of $\S 2$. As an approximation to the eigenvalue we take the difference

$$
\mathcal{L}_{1}^{\varepsilon}=\Lambda_{\dagger}-\varepsilon^{2} \mu^{\prime} \in\left(0, \Lambda_{\dagger}\right)
$$




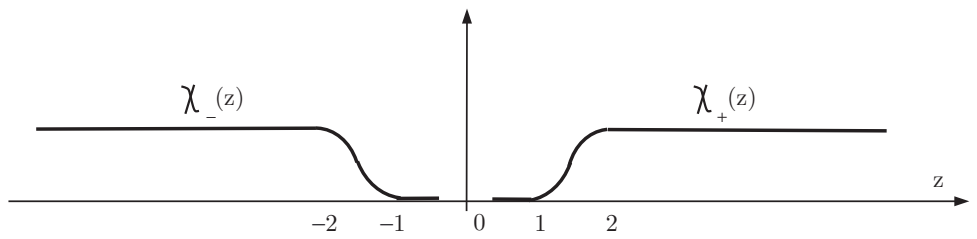

Figure 5. The cutoff functions $\chi_{ \pm}$.

where $\mu^{\prime}$ is the positive correction (2.29). The global approximation to the eigenfunction looks like this:

$$
\begin{aligned}
\mathcal{U}_{1}^{\varepsilon}(x)= & X_{1 / \varepsilon}(z)\left(w^{0}(x)+\varepsilon w^{\prime}(x)\right) \\
& +\sum_{ \pm} \chi_{ \pm}(z)\left(c_{ \pm}^{0}+\varepsilon c_{ \pm}^{\prime}\right) \exp \left(\mp \varepsilon z \sqrt{\mu^{\prime}}\right) U_{\dagger}(y) \\
& -\sum_{ \pm} \chi_{ \pm}(z) X_{1 / \varepsilon}(z)\left(c_{ \pm}^{0}+\varepsilon\left(c_{ \pm}^{\prime} \mp c_{ \pm}^{0} z \sqrt{\mu^{\prime}}\right)\right) U_{\dagger}(y)
\end{aligned}
$$

We have applied an asymptotic construction with overlapping cutoff functions (see 31] and also [7, §2.1] and [32]). The graphs of the cutoff functions

$$
\chi_{ \pm}(z)=\chi( \pm z-1), \quad X_{1 / \varepsilon}(z)=\chi(z+1 / \varepsilon) \chi(-z+1 / \varepsilon)
$$

are depicted in Figures 5 and 4 , where we should put $R=1 / \varepsilon$, and $\chi$ is the standard cutoff function introduced after (2.5). The coefficients $c_{ \pm}^{p}$ are given by identities (2.28), which ensure the correlation of (2.26) and (2.27). In the subtrahend (the last sum in (3.2)) we have collected all terms involved in correlation; in what follows, when we commute differential operators and the cutoff functions $\chi_{ \pm}$or $X_{1 / \varepsilon}$, this subtrahend will be absorbed (accordingly) by the first or the second term on the right in (3.2)), which will ensure the discrepancies being small.

Remark 3.1. If $H_{ \pm}>0$ (see Figure 1a), then the function $w^{\prime}$, given initially on $\Omega^{0}$, is defined also on the perturbed domain $\Omega^{\varepsilon} \subset \Omega^{0}$, but if the profile functions $H_{ \pm}$are arbitrary, then the inclusion $\Omega^{\varepsilon} \subset \Omega^{0}$ may fail (cf. Figure 1b), and the summand $w^{\prime}(x)$ needs interpretation. By Lemma 2.2, the solution $w^{\prime}$ of problem (2.20), (2.19) admits extension through the surfaces $\theta_{ \pm}^{0}$ of the screen $\Theta^{0}$ to the lenses $\boldsymbol{\Theta}_{ \pm}^{\varepsilon}=\left\{x \in \boldsymbol{\Theta}^{\varepsilon}: \mp z>0\right\}$; the parameter $h_{\Theta}>0$ can be chosen so that the biconvex lens $\Theta^{\varepsilon}=\left\{x \in \Omega^{0}: \rho(x)<c_{\Theta} \varepsilon\right\}$ includes the screen $\Theta^{\varepsilon}$. In the middle zone of the screen $\Theta^{0}$ we can do extensions, e.g., with the help of the Taylor formula, i.e.,

$$
\widehat{w}^{\prime}(y, z)=\sum_{j=0}^{N} \frac{z^{j}}{j !} \frac{\partial^{j} w^{\prime}}{\partial z^{j}}(y, \mp 0), \quad(y, z) \in \mathbf{\Theta}_{ \pm}^{\varepsilon} \backslash \mathcal{V} .
$$

Near the edge $\theta^{0}$, a similar Taylor formula is applied relative to the angle variable $\varphi \in(-\pi, \pi)$, where $(\rho, \varphi)$ is the system of polar coordinates on the planes orthogonal to the edge. Thus, using a cutoff functions $\chi_{\theta} \in C^{\infty}(\theta)$ that is equal to 1 near $\partial \theta$ and provides, together with $1-\chi_{\theta}$, a partition of unity on $\vartheta^{0}$, we obtain

$$
\widehat{w}^{\prime}(y, z)=\left(1-\chi_{\theta}(y)\right) \sum_{j=0}^{N} \frac{z^{j}}{j !} \frac{\partial^{j} w^{\prime}}{\partial z^{j}}(y, \mp 0)+\left.\sum_{j=0}^{N} \frac{(\phi \pm \pi)^{j}}{j !} \frac{\partial^{j}\left(\chi_{\theta} w^{\prime}\right)}{\partial \phi^{j}}(y, z)\right|_{\phi=\mp \pi} .
$$

It should be emphasized that we extend not to the cylinder $\Omega$ itself, but to some set not embedded in $\mathbb{R}^{n}$ (cf. Figure 6). For any shape of $\theta_{ \pm}^{\varepsilon}$, the extension $\widehat{w}^{\prime}$ is defined in the domain $\Omega^{\varepsilon}$; it is smooth outside the neighborhood $\mathcal{V}$ of the edge $\vartheta^{\varepsilon}=\vartheta^{0}$, and inside 


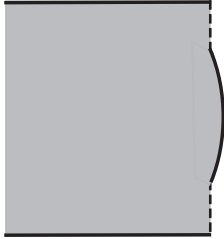

a

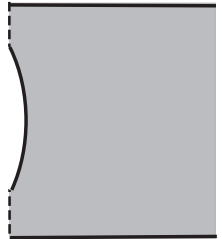

$\mathrm{b}$

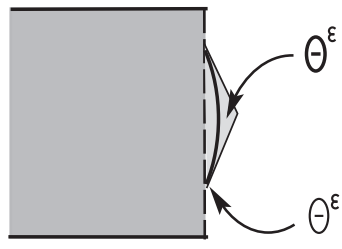

$\mathrm{C}$

FiguRE 6. Extension (c) from the left half (a) of the waveguide in Figure 1a; for the right half (b) no extension is needed.

$\mathcal{V}$ it still admits estimates like (2.43). Finally, by enlarging the number $N \in \mathbb{N}$ in the definition (3.4), we can arrange that equation (2.20) be preserved on each of the two lenses with an accuracy prescribed in advance:

$$
\left|\Delta_{x} w^{\prime}(x)+\Lambda_{\dagger} w^{\prime}(x)\right| \leq c \varepsilon^{N-2} \rho(x)^{-\delta-3 / 2} .
$$

2. Discrepancy estimate in the spectral problem. We want to estimate the discrepancy of the asymptotic approximations (3.2) and (3.1) in the variational problem (1.8):

$$
\begin{aligned}
\delta & =\sup \left|\left(\nabla_{x} \mathcal{U}_{1}^{\varepsilon}, \nabla_{x} v^{\varepsilon}\right)_{\Omega^{\varepsilon}}-\mathcal{L}_{1}^{\varepsilon}\left(\mathcal{U}_{1}^{\varepsilon}, v^{\varepsilon}\right)_{\Omega^{\varepsilon}}\right| \\
& =\sup \left|\left(\Delta_{x} \mathcal{U}_{1}^{\varepsilon}+\mathcal{L}_{1}^{\varepsilon} \mathcal{U}_{1}^{\varepsilon}, v^{\varepsilon}\right)_{\Omega^{\varepsilon}}-\sum_{ \pm}\left(\partial_{\nu} \mathcal{U}_{1}^{\varepsilon}, v^{\varepsilon}\right)_{\theta_{ \pm}^{\varepsilon}}\right|
\end{aligned}
$$

The supremum is taken over all $v^{\varepsilon} \in \stackrel{\circ}{H}^{1}\left(\Omega^{\varepsilon} ; \Gamma\right)$ such that $\left\|\nabla_{x} v^{\varepsilon} ; L_{2}\left(\Omega^{\varepsilon}\right)\right\|=1$.

Remark 3.2. By (1.12), we have

$$
\left\|v^{\varepsilon} ; L_{2}\left(\Omega^{\varepsilon}\right)\right\|+\sum_{ \pm}\left\|v^{\varepsilon} ; L_{2}\left(\theta_{ \pm}^{\varepsilon}\right)\right\| \leq C\left\|\nabla_{x} v^{\varepsilon} ; L_{2}\left(\Omega^{\varepsilon}\right)\right\|=C .
$$

As before, the constant $C$ depends neither on $v^{\varepsilon} \in \stackrel{\circ}{H}^{1}\left(\Omega^{\varepsilon} ; \Gamma\right)$, nor on $\varepsilon \in(0,1]$. The verification of (1.15) is standard. By the Friedrichs inequality (1.15) and a similar inequality on the domain $\omega \backslash \bar{\theta}$, integrated additionally over the variable $z$, the quantity $c\left\|\nabla_{x} v^{\varepsilon} ; L_{2}\left(\Omega^{\varepsilon}\right)\right\|^{2}$ dominates the $L_{2}$-norm of $v^{\varepsilon}$ on the union of the sets $\omega \times(-\infty,-R)$, $\omega \times(R,+\infty)$, and $(\omega \backslash \theta) \times(-R, R)$. Observe the simple one-dimensional inequality

$$
\left|v^{\varepsilon}\left(y, \varepsilon H_{+}(y)\right)\right|^{2}+\int_{\varepsilon H_{+}(y)}^{R}\left|v^{\varepsilon}(y, z)\right|^{2} d z \leq c\left(\int_{\varepsilon H_{+}(y)}^{2 R}\left|\frac{\partial v^{\varepsilon}}{\partial z}(y, z)\right|^{2} d z+\int_{R}^{2 R}\left|v^{\varepsilon}(y, z)\right|^{2} d z\right)
$$

with constant $c$ independent of $\varepsilon \leq 1$; after integration over $\omega \backslash \bar{\theta} \ni y$, it extends the $L_{2}$-estimate to the set $\left\{(y, z): y \in \theta, \varepsilon H_{+}(y)<z<R\right\}$ and, at the same time, provides the majorant in (3.7) for the norm $\left\|v^{\varepsilon} ; L_{2}\left(\theta_{+}^{\varepsilon}\right)\right\|$, because on the surface $\theta_{+}^{\varepsilon}$ the Jacobian indicated in (2.16) is bounded uniformly in $\varepsilon \leq 1$. It remains to repeat the last phrases for $H_{-}$in place of $H_{+}$. 
We return to the discrepancy (3.6). We have

$$
\begin{aligned}
\Delta_{x} \mathcal{U}_{1}^{\varepsilon}(x) & +\mathcal{L}_{1}^{\varepsilon} \mathcal{U}_{1}^{\varepsilon}(x)=X_{1 / \varepsilon}(z)(\underbrace{\Delta_{x} w^{0}(x)+\Lambda_{\dagger} w^{0}(x)}+\varepsilon(\underbrace{\Delta_{x} w^{\prime}(x)+\Lambda_{\dagger} w^{\prime}(x)})) \\
& +\varepsilon^{2} \mu^{\prime} X_{1 / \varepsilon}(z)\left(w^{0}(x)+\varepsilon w^{\prime}(x)-\sum_{ \pm} \chi_{ \pm}(z)\left(c_{ \pm}^{0}+\varepsilon\left(c_{ \pm}^{1} \mp c_{ \pm}^{0} z \sqrt{\mu^{\prime}}\right)\right) U_{\dagger}(y)\right) \\
& +\sum \pm \chi_{ \pm}(z)\left(c_{ \pm}^{0}+\varepsilon c_{ \pm}^{1}\right) \underbrace{\left(\Delta_{x}+\Lambda_{\dagger}-\varepsilon^{2} \mu^{\prime}\right)\left(\exp \left(\mp \varepsilon z \sqrt{\mu^{\prime}}\right) U_{\dagger}(y)\right)} \\
& +\left[\Delta_{x}, X_{1 / \varepsilon}(z)\right]\left(w^{0}(x)+\varepsilon w^{\prime}(x)-\left(c_{ \pm}^{0}+\varepsilon\left(c_{ \pm}^{1} \mp c_{ \pm}^{0} z \sqrt{\mu^{\prime}}\right)\right) U_{\dagger}(y)\right) \\
& +\sum_{ \pm}\left[\Delta_{x}, \chi_{ \pm}(z)\right]\left(\left(c_{ \pm}^{0}+\varepsilon c_{ \pm}^{1}\right) \exp \left(\mp \varepsilon z \sqrt{\mu^{\prime}}\right)-\left(c_{ \pm}^{0}+\varepsilon\left(c_{ \pm}^{1} \mp c_{ \pm}^{0} z \sqrt{\mu^{\prime}}\right)\right)\right) U_{\dagger}(y) \\
= & : I_{1}(x)+I_{2}(x)+I_{3}(x)+I_{4}(x)+I_{5}(x) .
\end{aligned}
$$

Here $\left[\Delta_{x}, X_{1 / \varepsilon}\right]$ and $\left[\Delta_{x}, \chi_{ \pm}\right]$are the commutators of the Laplace operator with cutoff functions, e.g.,

$$
\left[\Delta_{x}, \chi_{ \pm}(z)\right] f(x)=2 \partial_{z} f(x) \partial_{z} \chi_{ \pm}(z)+f(x) \partial_{z}^{2} \chi_{ \pm}(z) .
$$

We start with terms that involve commutators. By (3.3) and (3.8), the quantity $I_{4}(x)$ vanishes if $|z| \notin\left[\varepsilon^{-1}-1, \varepsilon^{-1}\right]$. Relations (2.28), (2.29), (1.17), and (2.21) imply that $I_{4}$ decays exponentially, i.e., for some positive $\gamma$ and $c$ we have

$$
\left|\left(I_{4}, v^{\varepsilon}\right)_{\Omega^{\varepsilon}}\right| \leq c \exp (-\gamma / \varepsilon)\left\|v^{\varepsilon} ; L_{2}\left(\Omega^{\varepsilon}\right)\right\| .
$$

Also, $I_{5}(x)=0$ for $|z| \geq 2$; consequently, by the Taylor formula for the exponential function, we get

$$
\left|\left(I_{5}, v^{\varepsilon}\right)_{\Omega^{\varepsilon}}\right| \leq c \varepsilon^{2}\left\|v^{\varepsilon} ; L_{2}\left(\Omega^{\varepsilon}\right)\right\| .
$$

Differentiation shows that the braced factor in $I_{3}$ vanishes. Since, in $I_{2}$, from the sum $w^{0}(x)+\varepsilon w^{\prime}(x)$ we subtracted the linear terms of the asymptotic expansion as $z \rightarrow \pm \infty$ (again, see (1.17), (2.21) and (2.28), (2.29) ), we have

$$
\left|\left(I_{2}, v^{\varepsilon}\right)_{\Omega^{\varepsilon}}\right| \leq c \varepsilon^{2}\left\|v^{\varepsilon} ; L_{2}\left(\Omega^{\varepsilon}\right)\right\| .
$$

Finally, in $I_{1}$ the first braced sum is always zero, but the second vanishes only if $H_{ \pm} \geq 0$, when the function $w^{\prime}$ needs no extension. If the inclusion $\Omega^{\varepsilon} \subset \Omega^{0}$ fails, then, by (3.5), we have

$$
\left|\left(I_{1}, v^{\varepsilon}\right)_{\Omega^{\varepsilon}}\right| \leq c \varepsilon^{N-2}\left(\int_{\Theta^{\varepsilon}} \rho(x)^{2 \delta-3} d x\right)^{1 / 2}\left\|\rho^{-2 \delta} v^{\varepsilon} ; L_{2}\left(\Omega^{\varepsilon} \cap \boldsymbol{\Theta}\right)\right\| c \varepsilon^{N-2}\left\|\nabla_{x} v^{\varepsilon} ; L_{2}\left(\Omega^{\varepsilon}\right)\right\| .
$$

The integral over the biconvex lens $\Theta$ converges because this lens is tapered angularly near the edge $\vartheta^{\varepsilon}$ (see the definition in Remark 3.1), and the (employed) estimate

$$
\left\|\rho^{-1}(1+|\ln \rho|)^{-1} v^{\varepsilon} ; L_{2}\left(\Omega^{\varepsilon}\right)\right\| \leq c\left\|v^{\varepsilon} ; H^{1}\left(\Omega^{\varepsilon}\right)\right\|
$$

for the weighted $L_{2}$-norm of the test function for $\delta<1 / 2$ is ensured by the well-known 1-dimensional Hardy inequality "with logarithm":

$$
\int_{0}^{1} \varrho^{-1}|\ln \varrho|^{-2}|V(\varrho)|^{2} d \varrho \leq 4 \int_{0}^{1} \varrho\left|\frac{d V}{d \varrho}(\varrho)\right|^{2} d \varrho, \quad V \in C_{c}^{1}[0,1) .
$$

Now we treat the last scalar products in (3.6). Recalling (2.17) and observing that $\mathcal{U}_{1}^{\varepsilon}=w^{0}+\varepsilon w^{\prime}$ in a neighborhood of the screen $\Theta^{\varepsilon}$ (because $\chi_{ \pm}(z)=0$ for $|z| \leq 1$ ), we obtain

$$
\begin{aligned}
\partial_{\nu} \mathcal{U}_{1}^{\varepsilon}\left(y, \pm \varepsilon H_{ \pm}(y)\right)= & {\left[1+\varepsilon^{2}\left|\nabla_{y} H_{ \pm}(y)\right|^{2}\right]^{-1 / 2}\left(\mp \partial_{z} w^{0}(y)+\varepsilon \nabla_{y} H_{ \pm}(y) \cdot \nabla_{y} w^{0}(y)\right.} \\
& \left.\mp \varepsilon \partial_{z} w^{\prime}\left(y, \pm \varepsilon H_{ \pm}(y)\right)+\varepsilon^{2} \nabla_{y} H_{ \pm}(y) \cdot \nabla_{y} w^{\prime}\left(y, \pm \varepsilon H_{ \pm}(y)\right)\right) .
\end{aligned}
$$


Consequently, we can use (2.19) and (2.44) to get

$$
\left|\left(\partial_{\nu} \mathcal{U}_{1}^{\varepsilon}, v^{\varepsilon}\right)_{\theta_{ \pm}^{\varepsilon}}\right| \leq c \varepsilon^{2}\left(\int_{\theta_{ \pm}^{\varepsilon}} \rho(x)^{-2(\delta+1 / 2} d s_{x}\right)^{1 / 2}\left\|v^{\varepsilon} ; L_{2}\left(\theta_{ \pm}^{\varepsilon}\right)\right\| \leq c \varepsilon^{2} .
$$

We have also used estimate (3.7) for the test function $v^{\varepsilon}$ and the convergence of integrals over the surfaces $\theta_{ \pm}^{\varepsilon}$.

Summarizing, we collect the inequalities (3.9)-3.12) obtained for the components on the right-hand side of (3.6); this yields the following estimate:

$$
\delta \leq c_{\delta} \varepsilon^{2} .
$$

The "worst" majorants occurred in (3.10), (3.11), and (3.12). At first glance, it seems that relation (3.13) does not suffice for justifying the asymptotic formulas for eigenvalues, constructed with the same accuracy $O\left(\varepsilon^{2}\right)$. Below it will be shown that this impression is wrong.

3. Spectral measure expansion. By the spectral theorem (see [3, Theorem 6.1.1]), the selfadjoint operator $A^{\varepsilon}$ in $L_{2}\left(\Omega^{\varepsilon}\right)$ introduced in Subsection 2 of $\S 24$ generates a spectral measure $\mathbf{E}_{A^{\varepsilon}}$ in $L_{2}\left(\Omega^{\varepsilon}\right)$, which, in its turn, assigns the complex measure $\boldsymbol{\mu}_{\mathbf{u}, \mathbf{v}}=$ $\left(\mathbf{E}_{A^{\varepsilon}} \mathbf{u}, \mathbf{v}\right)_{\Omega^{\varepsilon}}$ on $\mathbb{R}$ to each pair of elements $\mathbf{u}, \mathbf{v} \in L_{2}\left(\Omega^{\varepsilon}\right)$. Below we shall need some simple formulas (see, e.g., the proof of Theorem 6.1.3 in [3]), namely,

$$
(\mathbf{u}, \mathbf{v})_{\Omega^{\varepsilon}}=\int_{\mathbb{R}} d \boldsymbol{\mu}_{\mathbf{u}, \mathbf{v}}(\mathbf{t})
$$

and, for $\boldsymbol{\lambda} \in \mathbb{C}$ and $\mathbf{u}$ in the domain $\mathcal{D}\left(A^{\varepsilon}\right)$ of the operator $A^{\varepsilon}$,

$$
\left\|A^{\varepsilon} \mathbf{u}-\boldsymbol{\lambda} \mathbf{u} ; L_{2}\left(\Omega^{\varepsilon}\right)\right\|^{2}=\int_{\mathbb{R}}(\mathbf{t}-\boldsymbol{\lambda})^{2} d \boldsymbol{\mu}_{\mathbf{u}, \mathbf{u}}(\mathbf{t}) .
$$

Assume that, for some $\ell$ and $\alpha$ (generally speaking, large and small, respectively), the interval

$$
\Upsilon=\left(\Lambda_{\dagger}-\varepsilon^{2} \mu^{\prime}-\ell \varepsilon^{2+\alpha}, \Lambda_{\dagger}-\varepsilon^{2} \mu^{\prime}+\ell \varepsilon^{2+\alpha}\right)
$$

is free of the spectrum of $\left(A^{\varepsilon}\right)$ and that we have found an element $\mathbf{u} \in \mathcal{D}\left(A^{\varepsilon}\right)$ such that

$$
\left\|\mathbf{u} ; L_{2}\left(\Omega^{\varepsilon}\right)\right\|=1, \quad\left\|A^{\varepsilon} \mathbf{u}-\left(\Lambda_{\dagger}-\varepsilon^{2} \mu^{\prime}\right) \mathbf{u} ; L_{2}\left(\Omega^{\varepsilon}\right)\right\|=: \delta .
$$

Then relations (3.14) and (3.15) will imply

$$
\begin{aligned}
\delta^{2} & =\left\|A^{\varepsilon} \mathbf{u}-\left(\Lambda_{\dagger}-\varepsilon^{2} \mu^{\prime}\right) \mathbf{u} ; L_{2}\left(\Omega^{\varepsilon}\right)\right\|^{2}=\int_{\mathbb{R}}\left(\mathbf{t}-\Lambda_{\dagger}+\varepsilon^{2} \mu^{\prime}\right)^{2} d \boldsymbol{\mu}_{\mathbf{u}, \mathbf{u}}(\mathbf{t}) \\
& \geq \ell^{2} \varepsilon^{4+2 \alpha} \int_{\mathbb{R}} d \boldsymbol{\mu}_{\mathbf{u}, \mathbf{u}}(\mathbf{t}) \geq \ell^{2} \varepsilon^{4+2 \alpha}\left\|\mathbf{u} ; L_{2}\left(\Omega^{\varepsilon}\right)\right\|^{2}=\ell^{2} \varepsilon^{4+2 \alpha} .
\end{aligned}
$$

Recalling estimate (3.13) we put

$$
\mathbf{u}(x)=\left\|\mathcal{U}_{1}^{\varepsilon} ; L_{2}\left(\Omega^{\varepsilon}\right)\right\|^{-1} \mathcal{U}_{1}^{\varepsilon}(x), \quad \boldsymbol{\delta}=\left\|\mathcal{U}_{1}^{\varepsilon} ; L_{2}\left(\Omega^{\varepsilon}\right)\right\|^{-1} \delta \leq c_{\delta} \varepsilon^{2}\left\|\mathcal{U}_{1}^{\varepsilon} ; L_{2}\left(\Omega^{\varepsilon}\right)\right\|^{-1} .
$$

then the first condition in (3.17) is satisfied. We compute the $L_{2}$-norm of the global asymptotic approximation (3.2) and observe the inequality

$$
\left\|\mathcal{U}_{1}^{\varepsilon} ; L_{2}\left(\Omega^{\varepsilon}\right)\right\|^{2} \geq c_{\mathcal{U}} \varepsilon^{-1}, \quad c_{\mathcal{U}}>0,
$$

The last two formulas show that $\delta \leq \mathbf{c}_{\delta} \varepsilon^{5 / 2}$, so that (3.18) is absurd for $\alpha=1 / 2$ and sufficiently small $\ell>0$ and $\varepsilon>0$. Hence, our assumption that the interval (3.16) is free of the spectrum of $A^{\varepsilon}$ cannot be true: this interval $\Upsilon$, located below the continuous

\footnotetext{
${ }^{4}$ For the reader's convenience, we keep the notation used in the book [3], but write it in bold type letters.
} 
spectrum, must contain an eigenvalue. So, we have drawn the required conclusion; it remains to explain inequality (3.20). form

Regrouping the terms in (3.2), we write the global asymptotic approximation in the

$$
\mathcal{U}_{1}^{\varepsilon}=\varepsilon X_{1 / \varepsilon}\left(w^{1} \mp \sum_{ \pm} \chi_{ \pm}\left(b_{0}^{\prime}+b_{z}^{\prime}\right) U_{\dagger}\right)+\sum_{ \pm} \chi_{ \pm}\left(1 \pm \varepsilon b_{0}^{\prime}\right) \exp \left(\mp \varepsilon z \sqrt{\mu^{\prime}}\right) U_{\dagger} .
$$

Since the coefficient of $X_{1 / \varepsilon}$ decays exponentially at infinity, its Lebesgue norm is $O(1)$ as $\varepsilon \rightarrow+0$. We have

$$
\int_{2}^{\infty} \exp \left(-2 \varepsilon z \sqrt{\mu^{\prime}}\right) d z=\frac{1}{2 \varepsilon \sqrt{\mu^{\prime}}} \exp \left(-4 \varepsilon \sqrt{\mu^{\prime}}\right)
$$

and inequality (3.20) with a positive constant $c_{\mathcal{U}}$ becomes evident.

4. Eigenvalues below the continuous spectrum: asymptotic expansion and uniqueness. We start with the following simple statement.

Proposition 3.1. For small $\varepsilon>0$, the interval $\left(0, \Lambda_{\dagger}\right)$ can contain at most one eigenvalue of the operator $A^{\varepsilon}$ (of problem (1.8)).

Proof. Turning once again to problem (1.27) in the finite cylinder $\Omega_{L}^{\varepsilon}$ with a cavity (1.1), we note that the first eigenvalue $\tau_{1}^{0}=\Lambda_{\dagger}$ of the limit problem in $\Omega_{L}^{0}$ is simple (see, e.g., [2]), i.e., for the second eigenvalue we have $\tau_{2}^{0}>\Lambda_{\dagger}$. Since the eigenvalues of the perturbed problem satisfy $\tau_{k}^{\varepsilon}=\tau_{k}^{0}+O(\varepsilon)$ (cf. Proposition 1.1, and also the asymptotic formula in [7, Chapter 9 and $\S 5.5])$, for small $\varepsilon>0$ we have the inequality

$$
\tau_{2}^{\varepsilon} \geq \tau_{2}^{0}-\frac{1}{2}\left(\tau_{2}^{0}-\Lambda_{\dagger}\right)=\frac{1}{2}\left(\tau_{2}^{0}+\Lambda_{\dagger}\right)>\Lambda_{\dagger} .
$$

Consequently, if a function $u^{\varepsilon} \in \stackrel{\circ}{H}^{1}\left(\Omega^{\varepsilon} ; \Gamma\right)$ is subject to the orthogonality condition

$$
\left(u^{\varepsilon}, v_{1}^{\varepsilon}\right)_{\Omega_{L}^{\varepsilon}}=0 \text {, }
$$

where $v_{1}^{\varepsilon}$ is the first eigenfunction of problem (1.27), then

$$
\Lambda_{\dagger}\left\|u^{\varepsilon} ; L_{2}\left(\Omega_{L}^{\varepsilon}\right)\right\|^{2} \leq \tau_{2}^{\varepsilon}\left\|u^{\varepsilon} ; L_{2}\left(\Omega_{L}^{\varepsilon}\right)\right\|^{2} \leq\left\|\nabla_{x} u^{\varepsilon} ; L_{2}\left(\Omega_{L}^{\varepsilon}\right)\right\|^{2} .
$$

Now suppose that there are two discrete spectrum points on $\left(0, \Lambda_{\dagger}\right)$. By the maximin principle (see, e.g., [3, Theorem 10.2.2]), the second eigenvalue $\lambda_{2}^{\varepsilon}$ is given by the formula

$$
\lambda_{2}^{\varepsilon}=\max _{\mathcal{E}_{1}} \inf _{u^{\varepsilon} \in \mathcal{E}_{1} \backslash\{0\}} \frac{\left\|\nabla_{x} u^{\varepsilon} ; L_{2}\left(\Omega^{\varepsilon}\right)\right\|^{2}}{\left\|u^{\varepsilon} ; L_{2}\left(\Omega^{\varepsilon}\right)\right\|^{2}} .
$$

Here $\mathcal{E}_{1} \subset \stackrel{\circ}{H}^{1}\left(\Omega^{\varepsilon} ; \Gamma\right)$ is an arbitrary subspace of codimension 1 . For the role of $\mathcal{E}_{1}$ we take the subspace $\mathcal{E}_{1}^{\varepsilon}$ distinguished by (3.21). To inequality (3.22) (without its middle part), we add the relation obtained by integration over $z \in(-\infty,-L) \cup(L,+\infty)$ of the Friedrichs inequality (1.15); this yields

$$
\Lambda_{\dagger}\left\|u^{\varepsilon} ; L_{2}\left(\Omega^{\varepsilon}\right)\right\|^{2} \leq\left\|\nabla_{x} u^{\varepsilon} ; L_{2}\left(\Omega^{\varepsilon}\right)\right\|^{2}, \quad u^{\varepsilon} \in \mathcal{E}_{1}^{\varepsilon} .
$$

Thus, the Rayleigh ratio under the infimum sign in (3.23) is at least $\Lambda_{\dagger}$, whence $\lambda_{2}^{\varepsilon} \geq \Lambda_{\dagger}$, which is impossible. This contradiction completes the proof.

Remark 3.3. In the case where $J_{\dagger}^{\prime}(H)<0$ (cf. formulas (2.24) and (2.30) $)$, the asymptotic representation (1.29) of the first eigenvalue of problem (1.27) shows that $\tau_{1}^{\varepsilon}>\Lambda_{\dagger}$ provided $\varepsilon>0$ is small. Consequently, using the minimum (rather than max-min) principle (cf. formulas (1.31) and (3.23) ) and repeating the proof of Proposition 3.1 with minor modifications, we see that if $J_{\dagger}^{\prime}(H)<0$ and $\varepsilon>0$ is small, then the discrete spectrum of the variational problem (1.8) (or the boundary value problem (1.5)-(1.7) is empty. 
By Proposition 3.1] the eigenvalue of problem (1.8) found in the preceding section is the only point of the discrete spectrum of the operator $A^{\varepsilon}$.

Now we turn to the justification of the asymptotic expansions deduced in Subsections 2 and $3, \S 3$.

Theorem 3.1. Under condition (2.24), there exist positive quantities $\varepsilon_{1}$ and $c_{1}, C_{1}$ such that for any $\varepsilon \in\left(0, \varepsilon_{1}\right)$ the discrete spectrum of the variational spectral problem (1.8) (or (1.5)-(1.7) in the differential form) has a unique point, namely, the eigenvalue $\lambda_{1}^{\varepsilon}$ satisfying the inequality

$$
\left|\lambda_{1}^{\varepsilon}-\frac{\varepsilon^{2}}{4}\left(\int_{\theta} H(y)\left(\left|\nabla_{x} U_{\dagger}(y)\right|^{2}-\Lambda_{\dagger}\left|U_{\dagger}(y)\right|^{2}\right) d y\right)^{2}\right| \leq c_{1} \varepsilon^{5 / 2} .
$$

The corresponding eigenfunction $u_{1}^{\varepsilon} \in \stackrel{\circ}{H}^{1}\left(\Omega^{\varepsilon} ; \Gamma\right)$ normalized in $L_{2}\left(\Omega^{\varepsilon}\right)$ (a trapped mode) satisfies

$$
\left\|u_{1}^{\varepsilon}-\varepsilon^{1 / 2}\left(\mu^{\prime}\right)^{1 / 4} \exp \left(-\varepsilon|z| \sqrt{\mu^{\prime}}\right) U_{\dagger} ; H^{1}\left(\Omega^{\varepsilon}\right)\right\| \leq C_{1} \varepsilon^{1 / 2},
$$

where $\mu^{\prime}$ is the quantity (2.29) (see also (2.24) and the subtrahend in (3.24)), and $\left\{\Lambda_{\dagger}, U_{\dagger}\right\}$ is the first eigenpair for problem (1.10) on the cross section $\omega$, and the normalization condition (1.11) is fulfilled.

Proof. It remains to check the claim concerning the eigenfunction. We return to the notation of formulas (3.17). Let $\mathbf{v} \in L_{2}\left(\Omega^{\varepsilon}\right)$. We already know that on the ray $\left(-\infty, \Lambda_{\dagger}\right)$ the measure $\boldsymbol{\mu}_{\mathbf{u}, \mathbf{v}}$ is concentrated at the point $\lambda_{1}^{\varepsilon} \in\left(0, \Lambda_{\dagger}\right)$ and

$$
\int_{-\infty}^{\Lambda_{\dagger}} d \boldsymbol{\mu}_{\mathbf{u}, \mathbf{v}}(\mathbf{t})=\left(\mathbf{P}_{1}^{\varepsilon} \mathbf{u}, \mathbf{v}\right)_{\Omega^{\varepsilon}}
$$

where $\mathbf{P}_{1}^{\varepsilon}$ is the orthogonal projection onto the subspace spanned by the eigenfunction $u_{1}^{\varepsilon}$. We have

$$
\begin{aligned}
\| \mathbf{u} & -\mathbf{P}_{1}^{\varepsilon} \mathbf{u} ; L_{2}\left(\Omega^{\varepsilon}\right) \|=\sup \left|\left(\mathbf{u}-\mathbf{P}_{1}^{\varepsilon} \mathbf{u}, \mathbf{v}\right)_{\Omega^{\varepsilon}}\right|=\sup \left|\int_{\Lambda_{\dagger}}^{+\infty} d \boldsymbol{\mu}_{\mathbf{u}, \mathbf{v}}(\mathbf{t})\right| \\
& \leq \frac{c}{\varepsilon^{2}} \sup \left|\int_{\Lambda_{\dagger}}^{+\infty}\left(\mathbf{t}-\Lambda_{\dagger}+\varepsilon^{2} \mu^{\prime}\right) d \boldsymbol{\mu}_{\mathbf{u}, \mathbf{v}}(\mathbf{t})\right| \\
& \leq \frac{c}{\varepsilon^{2}} \sup \left(\int_{\mathbb{R}}\left(\mathbf{t}-\Lambda_{\dagger}+\varepsilon^{2} \mu^{\prime}\right)^{2} d \boldsymbol{\mu}_{\mathbf{u}, \mathbf{u}}(\mathbf{t})\right)^{1 / 2}\left(\int_{\mathbb{R}} d \boldsymbol{\mu}_{\mathbf{v}, \mathbf{v}}(\mathbf{t})\right)^{1 / 2} \\
& \leq c \varepsilon^{-2+(2+\alpha)}=c \varepsilon^{\alpha} .
\end{aligned}
$$

Here the supremum is taken over all $\mathbf{v} \in L_{2}\left(\Omega^{\varepsilon}\right)$ such that $\left\|\mathbf{v} ; L_{2}\left(\Omega^{\varepsilon}\right)\right\|=1$.

Let $\mathbf{P}_{1}^{\varepsilon} \mathbf{u}=\mathbf{c}_{1}^{\varepsilon} u_{1}^{\varepsilon}$. Using (3.26), we see that $\left|\mathbf{c}_{1}^{\varepsilon}-1\right| \leq c \varepsilon^{1 / 2}$, because the two functions involved are normalized. Hence, $\left\|\mathbf{u}-u_{1}^{\varepsilon} ; L_{2}\left(\Omega^{\varepsilon}\right)\right\| \leq c \varepsilon^{\alpha}$.

Now we pass to the notation of (3.19) and recall that $\alpha=1 / 2$ and (3.20) holds true. As a result, we obtain

$$
\left\|\left(\mathcal{N}_{1}^{\varepsilon}\right)^{-1} \mathcal{U}_{1}^{\varepsilon}-u_{1}^{\varepsilon} ; L_{2}\left(\Omega^{\varepsilon}\right)\right\| \leq c \varepsilon^{1 / 2}, \quad \mathcal{N}_{1}^{\varepsilon}=\left\|\mathcal{U}_{1}^{\varepsilon} ; L_{2}\left(\Omega^{\varepsilon}\right)\right\| .
$$

By (1.8) and (3.13), for the quantity (3.6) we have

$$
\begin{aligned}
& \left\|\nabla_{x}\left(\left(\mathcal{N}_{1}^{\varepsilon}\right)^{-1} \mathcal{U}_{1}^{\varepsilon}-u_{1}^{\varepsilon}\right) ; L_{2}\left(\Omega^{\varepsilon}\right)\right\|^{2} \\
& \quad=\Lambda_{\dagger}\left(\left(\mathcal{N}_{1}^{\varepsilon}\right)^{-1} \mathcal{U}_{1}^{\varepsilon},\left(\mathcal{N}_{1}^{\varepsilon}\right)^{-1} \mathcal{U}_{1}^{\varepsilon}-u_{1}^{\varepsilon}\right)_{\Omega^{\varepsilon}}-\lambda_{1}^{\varepsilon}\left(u_{1}^{\varepsilon},\left(\mathcal{N}_{1}^{\varepsilon}\right)^{-1} \mathcal{U}_{1}^{\varepsilon}-u_{1}^{\varepsilon}\right)_{\Omega^{\varepsilon}}+O\left(\varepsilon^{2}\right),
\end{aligned}
$$

and this allows us to easily estimate the difference $\left(\mathcal{N}_{1}^{\varepsilon}\right)^{-1} \mathcal{U}_{1}^{\varepsilon}-u_{1}^{\varepsilon}$ in the Sobolev norm. 
We transform formula (3.2) for the global asymptotic approximation as follows:

$$
\begin{aligned}
\mathcal{U}_{1}^{\varepsilon}(x)- & \exp \left(-\varepsilon|z| \sqrt{\mu^{\prime}}\right) U_{\dagger}(y) \\
=(1 & \left.-\chi_{+}(z)-\chi_{-}(z)\right)\left(1-\exp \left(-\varepsilon|z| \sqrt{\mu^{\prime}}\right)\right) U_{\dagger}(y) \\
& +\varepsilon X_{1 / \varepsilon}(z)\left(w^{\prime}(x)+\sum_{ \pm} \mp \chi_{ \pm}(z)\left(b_{0}^{\prime}+b_{1}^{\prime} z\right) U_{\dagger}(y)\right) \\
& +\varepsilon \sum_{ \pm} \chi_{ \pm}(z) c_{ \pm}^{1} \exp \left(-\varepsilon|z| \sqrt{\mu^{\prime}}\right) U_{\dagger}(y) .
\end{aligned}
$$

Analyzing each group of terms on the right-hand side, we see that their norms in $H^{1}\left(\Omega^{\varepsilon}\right)$ do not exceed $c \varepsilon^{1 / 2}$. Finally, we have the obvious relation

$$
\left\|\exp \left(-\varepsilon|z| \sqrt{\mu^{\prime}}\right) U_{\dagger} ; L_{2}\left(\Omega^{\varepsilon}\right)\right\|=\varepsilon^{-1 / 2}\left(\mu^{\prime}\right)^{-1 / 4}+O\left(\varepsilon^{1 / 2}\right),
$$

completing the verification of estimate (3.25).

In the situation of (2.30), the justification of asymptotic formulas follows the same lines. Nevertheless, now the terms of the Ansatz (2.31) found in Subsection 4 of $\S 2$ do not suffice: we need to know the term $\varepsilon^{3} w^{\prime \prime \prime}(x)$. Due to the assumption (2.32) about the eigenvalue $\lambda^{\varepsilon}$, the function $w^{\prime \prime \prime}$ still satisfies the Helmholtz equation and the Dirichlet boundary conditions on $\Gamma$ (see (2.34)), and the Neumann boundary conditions (2.35) for $w^{\prime \prime \prime}$ are found with the help of calculations similar to (2.33), but taking the $O\left(\varepsilon^{3}\right)$ terms into account. These conditions look like this:

$$
\begin{gathered}
\mp \partial_{z} w^{\prime \prime \prime}(y, \pm 0)=g^{\prime \prime \prime}(y):=-\nabla_{y} H_{ \pm}(y) \cdot \nabla_{y}\left(w^{\prime \prime}(y, \pm 0) \pm H_{ \pm}(y) \partial_{z} w^{\prime}(y, \pm 0)\right) \\
-H_{ \pm}(y)\left(\partial_{z}^{2} w^{\prime \prime}(y, \pm 0) \pm \frac{1}{2} H_{ \pm}(y) \partial_{z}^{3} w^{\prime}(y, \pm 0)\right), \quad y \in \theta .
\end{gathered}
$$

The behavior of the functions $g^{\prime \prime \prime}$ as $y \rightarrow \partial \theta$, as guaranteed by Lemma 2.2. allows us to find a unique solution $w^{\prime \prime \prime} \in H_{\text {loc }}^{1}\left(\Omega^{0}\right)$ for which formula (2.36) is valid with the new coefficient $b_{0}^{\prime \prime \prime}$ and $b_{1}^{\prime \prime \prime}$ (see the next section). The asymptotic approximation is constructed with regard to the term $\varepsilon^{3} w^{\prime \prime \prime}(x)$, but no new arguments are required. The same can be said about the asymptotic justification procedure. We state the result.

Theorem 3.2. Under condition (2.30), there exist positive quantities $\varepsilon_{1}$ and $c_{1}, C_{1}$ such that for any $\varepsilon \in\left(0, \varepsilon_{1}\right)$ the discrete spectrum of the variational spectral problem (1.8) (or (1.5) -(1.7) in the differential form) has a unique point (namely, the eigenvalue $\left.\lambda_{1}^{\varepsilon}\right)$ satisfying the inequality

$$
\left|\lambda_{1}^{\varepsilon}-\Lambda_{\dagger}+\varepsilon^{4} \mu^{\prime \prime}\right| \leq c_{1} \varepsilon^{5}
$$

where $\mu^{\prime \prime}$ is the quantity that can be found by formulas (2.42), (2.40) starting with the bounded solution $w^{\prime}$ of problem (2.20), (2.19). The eigenfunction $u_{1}^{\varepsilon} \in \stackrel{\circ}{H}^{1}\left(\Omega^{\varepsilon} ; \Gamma\right)$ corresponding to $\lambda_{1}^{\varepsilon}$, normalized in $L_{2}\left(\Omega^{\varepsilon}\right)$ and representing a trapped mode, satisfies the inequality

$$
\left\|u_{1}^{\varepsilon}-\varepsilon\left(\mu^{\prime \prime}\right)^{1 / 4} \exp \left(-\varepsilon^{2}|z| \sqrt{\mu^{\prime \prime}}\right) U_{\dagger} ; H^{1}\left(\Omega^{\varepsilon}\right)\right\| \leq C_{1} \varepsilon^{1 / 2},
$$

where $\left\{\Lambda_{\dagger}, U_{\dagger}\right\}$ is the first eigenpair for problem (1.10) on the cross section $\omega$, and the normalization condition (1.11) is fulfilled.

5. Remarks on lower order asymptotic terms. The method of compound asymptotic expansions allows one to have no serious difficulties in finding the full asymptotic expansions of eigenvalues and eigenfunctions (to a certain extent, this was demonstrated in Subsections 4 of $\S 2$ and $\S 3$ ). The long computations (2.22) and (2.38), (2.39) were caused only by the necessity to find the sign of the eigenvalue increment and the desire to get more or less explicit formulas. Together with this, the Ansätze (2.15) and (2.31) 
have required additional work because their components do not decay (see Subsection 3 of $\S 2$ ). Now we discuss two technical tricks that can help to construct lower order terms of the asymptotic expression.

In the case of (2.31), when the Ansatz (2.31) is completed with the term $\varepsilon^{3} w^{\prime \prime \prime}(x)$, we get the formula

$$
\begin{aligned}
w^{0}(x) & +\varepsilon w^{\prime}(x)+\varepsilon^{2} w^{\prime \prime}(x)+\varepsilon^{3} w^{\prime \prime \prime}(x) \\
& =U_{\dagger}(y)\left(1 \pm \varepsilon b_{0}^{\prime} z \pm \varepsilon^{2}\left(b_{0}^{\prime \prime}+b_{1}^{\prime \prime} z\right) \pm \varepsilon^{3}\left(b_{0}^{\prime \prime \prime}+b_{1}^{\prime \prime \prime} z\right)\right)+\cdots .
\end{aligned}
$$

Next, taking the elongated Ansatz $\lambda^{\varepsilon}=\Lambda_{\dagger}-\varepsilon^{4} \mu^{\prime \prime}-\varepsilon^{5} \mu^{\prime \prime \prime}+\cdots$ into account, we obtain

$$
\begin{aligned}
& c_{ \pm}^{\varepsilon} U_{\dagger}(y) \exp \left(-|z| \sqrt{\Lambda_{\dagger}-\lambda^{\varepsilon}}\right) \\
& \quad=\left(c_{ \pm}^{0}+\varepsilon c_{ \pm}^{\prime}+\varepsilon^{2} c_{ \pm}^{\prime \prime}+\varepsilon^{3} c_{ \pm}^{\prime \prime \prime}+\cdots\right) U_{\dagger}(y) \exp \left(-|z| \sqrt{\varepsilon^{4} \mu^{\prime \prime}+\varepsilon^{5} \mu^{\prime \prime \prime}+\cdots}\right) \\
& \quad=U_{\dagger}(y)\left(c_{ \pm}^{0}+\varepsilon c_{ \pm}^{\prime}+\varepsilon^{2}\left(c_{ \pm}^{\prime \prime}-|z| \sqrt{\mu^{\prime \prime}}\right)+\varepsilon^{3}\left(c_{ \pm}^{\prime \prime \prime}-|z| \frac{\mu^{\prime \prime \prime}}{2 \mu^{\prime \prime}}\right)\right)+\cdots .
\end{aligned}
$$

These expansions can be correlated easily; as a result, in addition to (2.41) and (2.42), we find

$$
c_{ \pm}^{\prime \prime \prime}= \pm b_{0}^{\prime \prime \prime}, \quad b_{1}^{\prime \prime \prime}=-\frac{1}{2} \frac{\mu^{\prime \prime \prime}}{\mu^{\prime \prime}} \quad \Rightarrow \quad \mu^{\prime \prime \prime}=-2 \mu^{\prime \prime} b_{1}^{\prime \prime \prime} .
$$

In other words, the linear growth at infinity, which may potentially occur for the solution $w^{\prime \prime \prime}$ of problem (2.34), (3.27), is compensated by the next term $\varepsilon^{5} \mu^{\prime \prime \prime}$ of the Ansatz (2.32).

Now we describe how the term $-\varepsilon^{3} \mu^{\prime \prime \prime}$ of the Ansatz (2.25) can be taken into account, under the requirement (2.24). Because of the main correction $-\varepsilon^{2} \mu^{\prime \prime}$, the next term $\varepsilon^{2} w^{\prime \prime}(x)$ of (2.15) must satisfy the nonhomogeneous Helmholtz equation

$$
-\Delta_{x} w^{\prime \prime}(x)+\Lambda_{\dagger} w^{\prime \prime}(x)=-\mu^{\prime} w^{0}(y), \quad x \in \Omega^{0} .
$$

In (3.28), we collected and equated to zero the coefficients of $\varepsilon^{2}$ that arise when we plug the expansions $u^{\varepsilon}=w^{0}+\varepsilon w^{\prime}+\varepsilon^{2} w^{\prime \prime}+\cdots$ and $\lambda^{\varepsilon}=\Lambda_{\dagger}-\varepsilon^{2} \mu^{\prime \prime}-\varepsilon^{3} \mu^{\prime \prime \prime}+\cdots$ into equation (1.5). The nonhomogeneity $-\mu^{\prime} w^{0}(x)=-\mu^{\prime} U_{\dagger}(y)$ is compensated by the quadratic function

$$
\frac{1}{2} z^{2} \mu^{\prime} U_{\dagger}(y)
$$

which, luckily, satisfies the homogeneous Neumann conditions on the surfaces $\theta_{ \pm}^{0}$. Thus, we have

$$
\begin{aligned}
& w^{0}(x)+\varepsilon w^{\prime}(x)+\varepsilon^{2} w^{\prime \prime}(x) \\
& =U_{\dagger}(y)\left(1 \pm \varepsilon\left(b_{0}^{\prime}+b_{1}^{\prime} z\right)+\varepsilon^{2}\left(\frac{1}{2} z^{2} \mu^{\prime \prime} \pm b_{0}^{\prime \prime} \pm b_{1}^{\prime \prime} z\right)\right)+\cdots \\
& c_{ \pm}^{\varepsilon} U_{\dagger}(y) \exp \left(-|z| \sqrt{\Lambda_{\dagger}-\lambda^{\varepsilon}}\right) \\
& \quad=\left(c_{ \pm}^{0}+\varepsilon c_{ \pm}^{\prime}+\varepsilon^{2} c_{ \pm}^{\prime \prime}+\cdots\right) U_{\dagger}(y) \exp \left(-|z| \sqrt{\varepsilon^{2} \mu^{\prime}+\varepsilon^{3} \mu^{\prime \prime}+\cdots}\right) \\
& =U_{\dagger}(y)\left(c_{ \pm}^{0}+\varepsilon^{1}\left(c_{ \pm}^{\prime}-|z| \sqrt{\mu^{\prime}}\right)+\varepsilon^{2}\left(c_{ \pm}^{\prime \prime}-|z| \frac{\mu^{\prime \prime}}{2 \mu^{\prime}}+\frac{1}{2} \mu^{\prime}|z|^{2}\right)\right)+\cdots
\end{aligned}
$$

As a result, comparing the expansions indicated, in addition to (2.28) and (2.29) we get the formulas

$$
c_{ \pm}^{\prime \prime}= \pm b_{0}^{\prime \prime}, \quad b_{1}^{\prime \prime}=-\frac{1}{2} \frac{\mu^{\prime \prime}}{\mu^{\prime}} \quad \Rightarrow \quad \mu^{\prime \prime}=-2 \mu^{\prime} b_{1}^{\prime \prime} .
$$

Equally easy arguments allow us to construct the full asymptotic expansions for the eigenvalue and the eigenfunction. The justification of asymptotic formulas is done along the same lines as before. 


\section{§4. Other GEOMETRIC SHAPES}

1. Several screens. Quite obviously, the results obtained above generalize to the case of a disconnected open set $\theta$ (several screens; see Figure 3), and all asymptotic formulas need no substantial changes if we keep relations (1.3) on the entire smooth boundary $\partial \omega$.

2. A penny-shaped screen. For the screen

$$
\Theta^{\varepsilon}=\left\{(y, z): y \in \bar{\theta} \subset \mathbb{R}^{2},|z| \leq \varepsilon / 2\right\} \subset \mathbb{R}^{3}
$$

(see Figure 7a), both profile functions $H_{ \pm}$are constants, and the right-hand sides of the boundary condition (2.19) are zero. Therefore, at first glance, formula (2.29) for $\mu^{\prime}$ seems to become wrong. This impression is false. The point is that, because of the violation of condition (1.3), the boundary layer phenomenon arises near the edge of the coin, described via the solutions of the Neumann problem on the plane with the cut half-strip $\Pi=\left\{\xi=\left(\xi_{1}, \xi_{2}\right): \xi_{1} \geq 0,\left|\xi_{2}\right| \leq 1 / 2\right\}$ (Figure 8a), namely,

$$
\begin{aligned}
& -\Delta_{\xi} Z(\xi)=F(\xi), \quad \xi \in \Xi=\mathbb{R}^{2} \backslash \bar{\Pi}, \\
& \partial_{\nu} Z(\xi)=G(\xi), \quad \xi \in \partial \Xi .
\end{aligned}
$$

The domain $\Xi$ is obtained as a result of the variable change

$$
x \mapsto(\tau, \varsigma, z) \mapsto(\tau, \xi), \quad \xi=\left(\varepsilon^{-\alpha} \varsigma, \varepsilon^{-\alpha} z\right),
$$

where $\alpha=1,(\tau, \varsigma)$ is a curvilinear coordinate system near the contour $\partial \theta \subset \mathbb{R}^{2}, \tau$ is the arclength on the contour, and $\varsigma$ is the oriented distance to that contour, $\varsigma<0$ outside $\theta$. Note that $\Delta_{x}+\Lambda_{\dagger}=\varepsilon^{-2} \Delta_{\xi}+\cdots$, so that equation (4.2) and the boundary condition (4.3) are inherited from the initial relations (1.5) and (1.7).

As was already mentioned repeatedly, the function (1.17) satisfies the homogeneous Neumann conditions on the planes orthogonal to the $z$-axis; however, in the case under consideration, this function leaves the discrepancy $\partial_{\varsigma} U_{\dagger}(y)$ on the edge of the coin $\{x$ : $y \in \partial \theta,|z|<\varepsilon / 2\}$, that should be compensated for with the help of the solution of the problem with the right-hand sides $F=0$ and $G\left(\xi_{2}\right)=-\partial_{\varsigma} U_{\dagger}(\tau, 0)$ (we have put $\varsigma=0$ and $\xi_{2}=0$, but still view $\tau \in \partial \theta$ as a parameter; the smoothness of the boundary $\partial \theta$, i.e., smoothness relative to $\tau$, is required). No solution of the Neumann problem (4.2), (4.3) with such data possesses the inherent feature of a boundary layer, namely, the decay at infinity. There is a solution with the following behavior at infinity (see, e.g., the introductory chapter in [25]):

$$
Z(\xi ; \tau)=\frac{1}{2 \pi} \partial_{\varsigma} U_{\dagger}(\tau, 0) \ln |\xi|+O\left(|\xi|^{-1}\right), \quad|\xi| \rightarrow \infty .
$$

To avoid this contradiction, we allow for a logarithmic growth of the solution of equation (2.20) with the homogeneous boundary conditions (2.19) on the edge of the screen $\Theta^{0}$ :

$$
w^{\prime}(x)=\frac{1}{2 \pi} \partial_{\varsigma} U_{\dagger}(\tau, 0) \ln \varrho(x)+O(1), \quad x \rightarrow \vartheta^{0} ;
$$

here $\varrho(x)=\operatorname{dist}\left(x, \vartheta^{0}\right)$. In the framework of the compound expansions method [7, the singular solution $w^{\prime}$ in the Ansatz (2.15) should be multiplied by a cutoff function equal to zero in a $h$-neighborhood of the edge $\vartheta^{0}$ and to 1 outside the $2 h$-neighborhood of $\vartheta^{0}$. The extra discrepancy arising on the right in (4.2) due to commutation with the cutoff function, already taken into account when forming the function $F$, will ensure a powerlike decay of the solution $Z$. It should be mentioned that in the alternative method, that of matching expansions [33], the solution (4.5) itself serves as a term of the inner expansion, but in no way is it a boundary layer.

The singular solutions of such kind were studied in detail (see, e.g., [34, 35] and [25. Chapter 1]), and the homogeneous problem (2.20), (2.19) has a unique solution 
a

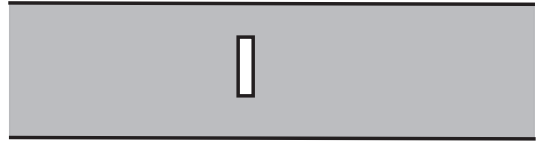

C

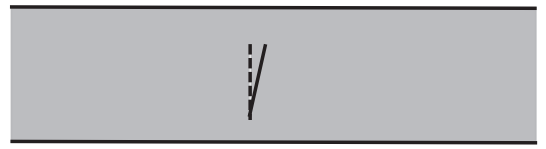

$\mathrm{b}$

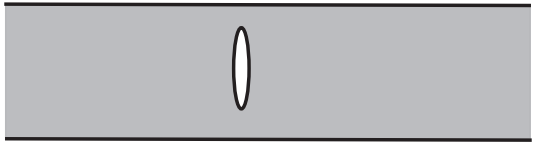

$\mathrm{d}$

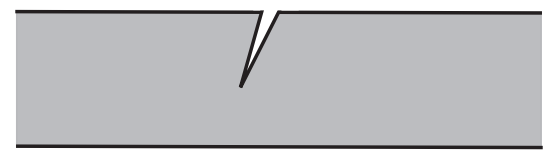

Figure 7
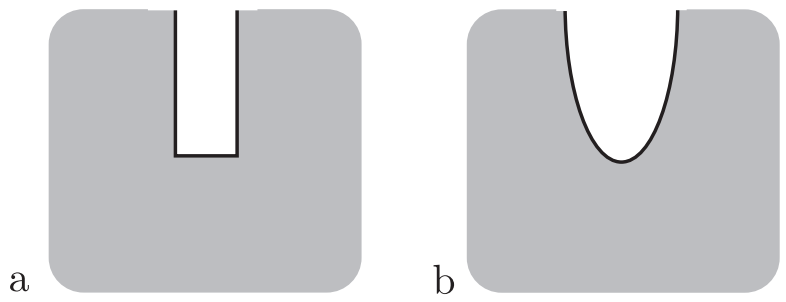

\section{FiguRE 8}

satisfying (4.6) and (2.21). The coefficient $b_{1}^{\prime}$ of the linear term is still calculated via the method of [22], but now the Green formula should be applied in the domain $\left\{x \in \Omega^{0}\right.$ : $|z|<R, \varrho(x)>1 / R\}$, and then we must pass to the limit as $R \rightarrow+\infty$. As a result, we obtain the identity

$$
b_{1}^{\prime}=-\int_{\partial \theta} U_{\dagger}(y) \partial_{\varsigma} U_{\dagger}(y) d \tau=-\int_{\theta}\left(\left|\nabla_{y} U_{\dagger}(y)\right|^{2}-\Lambda_{\dagger}\left|U_{\dagger}(y)\right|^{2}\right) d y,
$$

which does not differ from (2.22), because $H=1$ for the screen (4.1).

3. Screen with a smoothed edge. Let $n=2$, let $\Omega=\mathbb{R} \times(-1 / 2,1 / 2)$, and let $\Theta^{\varepsilon}$ be a thin ellipse,

$$
\Theta^{\varepsilon}=\left\{(y, z): \varepsilon^{-2} z^{2}+R^{-2}(y-L)^{2} \leq 1\right\},
$$

such that $-1 / 2<L-R<L+R<1 / 2$. Conditions (1.2) and (1.3) are fulfilled, but the profile functions $H_{ \pm}$(cf. formulas (4.7) and (1.1) ) become, first, smoothly dependent on the small parameter $\varepsilon$, and second, they lose smoothness near the points $Y_{ \pm}=L \pm R$ : their derivatives become singular. The first fact, in essence, does not influence the construction of asymptotic formulas: after application of the Taylor formula relative to $\varepsilon$, only simple modifications of the above calculations are required, but the second leads to substantial changes in asymptotic procedures, because the boundary layer phenomenon arises near the ends of the screen (cf. Subsection 2, §4).

In a neighborhood of the point $\left(Y_{-}, 0\right)$, we make a change of variables similar to (4.4):

$$
(y, z) \mapsto \xi=\left(\xi_{1}, \xi_{2}\right)=\left(\varepsilon^{-\alpha}(y-L+R), \varepsilon^{-\alpha} z\right) .
$$

Next, we rewrite the equation of the ellipse as follows:

$$
\begin{aligned}
\varepsilon^{2 \alpha} \xi_{2}^{2} & =z^{2}=\varepsilon^{2}\left(1-R^{-2}(y-L)^{2}\right) \\
& =\varepsilon^{2} R^{-2}\left(R^{2}-\left(\varepsilon^{\alpha} \xi_{1}-R\right)^{2}\right)=2 \varepsilon^{2+\alpha} R^{-1} \xi_{1}-\varepsilon^{2+2 \alpha} R^{-2} \xi_{1}^{2} .
\end{aligned}
$$

We discard the last summand (which is small for large $R$ and bounded $\xi_{1}$ ) and observe that, for $\alpha=2$, the small factor $\varepsilon^{4}$ in the two parts of (4.9) cancels; this leads to the 
following equation for the parabola $\Pi$ :

$$
\xi_{2}=2 R^{-1} \xi_{1}
$$

We see that the boundary layer has a narrower action domain, compared to the case of a "coin" (in Subsection 2 of $\S 4$ the dilation coefficient was equal to $\varepsilon^{-1}$ rather than to $\varepsilon^{-2}$ ), and is described with the help of solutions of problem (4.2), (4.3) on the plane from which the interior domain of a parabola is removed (Figure 8b).

For the first time, the above observations were made in the paper [36], where the matching expansions method was applied to construct the asymptotic formulas for the solution of the Dirichlet problem for the second order elliptic equation in a domain with a thin ellipse slit (see [33, and also $§ 5.5$ in the book [7], where more general boundary value problems for elliptic systems were treated). Nothing new arises when we pass to spectral problems with Neumann boundary conditions. Therefore, we restrict ourselves to saying that the claim similar to Theorem 3.1 is valid. Observe that, first, the restriction (2.24) is fulfilled, and second, the right-hand sides of the boundary condition in problem (2.20), (3.27) for $w^{\prime \prime}$ have singularities $O\left(\rho(x)^{-1}\right)$ at the ends of the segment $\theta=(L-R, L+R)$, i.e., the solution $w^{\prime \prime}$ can acquire logarithmic singularities (cf. (4.6)). Simple calculations (see, e.g., the introductory chapters in [7, 25]) show that in the case of the ellipse (4.7) the solution remains bounded near the screen $\Theta^{0}=\{(y, z): y \in[L-R, L+R], z=0\}$, but does not fall into the space $H_{\text {loc }}^{1}\left(\Omega^{0}\right)$, i.e., the Dirichlet integral diverges. In the case of the half-ellipse $\Theta^{\varepsilon}=\left\{(y, z): \varepsilon^{-2} z^{2}+R^{-2}(y-L)^{2} \leq 1, z \geq 0\right\}$, the solution $w^{\prime \prime}$ has a singularity $O\left(|\ln \rho|^{2}\right)$. However, all disorders disappear after the boundary layer is studied.

Note that if $m \in \mathbb{N}$ is even, then the boundary of the screen

$$
\Theta^{\varepsilon}=\left\{(y, z): \varepsilon^{-2 m} z^{2 m}+R^{-2 m}(y-L)^{2 m} \leq 1\right\}
$$

is smooth as before, and the boundary layer is described in the dilated coordinates $\xi$ from formula (4.8) with the exponent $\alpha=2 \frac{m}{m+1}$. It is not hard to find geometric conditions under which all tricks mentioned above work also for many-dimensional waveguides $\Omega^{\varepsilon}$.

4. A rotated rectilinear screen. The simplest way to perturb a rectilinear screen $\Theta^{0}$ of zero thickness is to rotate it by a small angle. Consider the corresponding 2-dimensional problem (1.5)-(1.7), i.e., let $n=2$, let $\omega=(-1 / 2,1 / 2)$, and let $\Omega=$ $\mathbb{R} \times(-1 / 2,1 / 2)$ be a strip of unit width (scaling has been applied). Also, let

$$
\begin{aligned}
& \Theta^{0}=\left\{x=(y, z): y \in\left[-L_{-}, L_{+}\right], z=0\right\}, \quad-1 / 2<-L_{-}<L_{+}<1 / 2, \\
& \Theta^{\varepsilon}=\left\{(y, z): y \in\left[-L_{-},-L_{-}+\left(1+\varepsilon^{2}\right)^{-1 / 2} L\right], z=\varepsilon\left(y+L_{-}\right)\right\} .
\end{aligned}
$$

It should be noted that the lengths of the segments (4.10) are equal to $L=L_{-}+L_{+}>0$, and $H_{ \pm}(y)= \pm\left(y+L_{-}\right)$, but the projection of $\Theta^{\varepsilon}$ to the y-axis is of length $\left(1+\varepsilon^{2}\right)^{-1 / 2} L=$ $L\left(1+O\left(\varepsilon^{2}\right)\right)$.

The increment of the projection length by the quantity $O\left(\varepsilon^{2}\right)$ does not effect the terms written in (2.31), in particular, does not affect $w^{\prime \prime}$, because $w^{0}=U_{\dagger}$ is the solution of problem (1.13), (1.14) whatever be the length of the rectilinear screen orthogonal to the $z$-axis.

Observe that

$$
U_{\dagger}(y)=\sqrt{2} \sin (\pi(y+1 / 2)), \quad \Lambda_{\dagger}=\pi^{2} .
$$

The right-hand side of the boundary condition (2.19) is equal to $-\pi \sqrt{2} \cos (\pi(y+1 / 2))$, which is a smooth function on the segment $\left.\left[-L_{-}, L_{+}\right]\right)$. Therefore, the solution $w^{\prime} \in$ $H_{\mathrm{loc}}^{1}\left(\Omega^{0}\right)$ admits the representation

$$
w^{\prime}(x)=\chi_{+}(x) K r_{+}^{1 / 2} \sin \left(\varphi_{+} 2\right)+\widehat{w}^{\prime}(x), \quad \widehat{w}^{\prime} \in H_{\mathrm{loc}}^{2}\left(\Omega^{0}\right)
$$


(see, e.g., Chapter 2 in 25]); here $K$ is some coefficient, $\left(r_{+}, \varphi_{+}\right)$is the polar coordinate system centered at the point $\left(L_{+}, 0\right), \varphi \in(-\pi, \pi)$, and $\chi_{+}$is a smooth cutoff function with small support and equal to 1 near this point. Because of the singularity $O\left(r_{+}^{-3 / 2}\right)$ of $\partial_{z}^{2} w^{\prime}$, problem (2.34), (2.35) has no solution $w^{\prime \prime}$ of class $H_{\mathrm{loc}}^{1}\left(\Omega^{0}\right)$. However, the results of [25, Chapter 2] show again that there is a solution looking like this:

$$
w^{\prime \prime}(x)=\chi_{+}(x) \frac{L}{2} K r_{+}^{-1 / 2} \cos \frac{\varphi_{+}}{2}+\widehat{w}^{\prime \prime}(x), \quad \widehat{w}^{\prime \prime} \in H_{\mathrm{loc}}^{1}\left(\Omega^{0}\right),
$$

and the first term is $O\left(r_{+}^{-1 / 2}\right)$, compensating for the singularity $O\left(r_{+}^{-3 / 2}\right)$ of the righthand side. As before, this solution satisfies (2.36) for any $d>0$. The coefficient $b^{\prime}$ can be found via the method of [22, but unlike the computations in (2.22) and (2.37), the Green formula is applied in the domain $\left\{x \in \Omega^{0}:|z|<R, r_{+}>\varrho\right\}$; this is a rectangle of size $1 \times 2 R$ from which a disk of a small radius $\varrho$, centered at $\left(L_{+}, 0\right)$, is cut off.

By (4.12), the extra integral

$$
\begin{aligned}
\int_{\left\{x: r_{+}=\varrho\right\}}\left(U_{\dagger} \frac{\partial w^{\prime \prime}}{\partial r_{+}}-w^{\prime \prime} \frac{\partial U_{\dagger}}{\partial r_{+}}\right) d s & =-U_{\dagger}\left(L_{+}\right) \frac{L}{4} K \varrho^{-3 / 2} \varrho \int_{-\pi}^{\pi} \cos \frac{\varphi_{+}}{2} d \varphi_{+}+O\left(\varrho^{1 / 2}\right) \\
& =-U_{\dagger}\left(L_{+}\right) L K \varrho^{-1 / 2}+O\left(\varrho^{1 / 2}\right)
\end{aligned}
$$

has no limit as $\varrho \rightarrow+0$. A similar growing summand (but with the plus sign) arises when we integrate by parts along the sides of the screen (cf. the first identity in (2.38) $)$, namely,

$$
\begin{aligned}
\sum_{ \pm} \pm & \int_{-L_{-}}^{L_{+}-\varrho} U_{\dagger}(y)\left(\partial_{y} w^{\prime}(y, \pm 0)+\left(y+L_{-}\right)\left(\partial_{y}^{2} w^{\prime}(y, \pm 0)+\Lambda_{\dagger} w^{\prime}(y, \pm 0)\right)\right) d y \\
= & \sum_{ \pm} \pm \int_{-L_{-}}^{L_{+}-\varrho} w^{\prime}(y, \pm 0)\left(\left(y+L_{-}\right)\left(\partial_{y}^{2} U_{\dagger}(y)+\Lambda_{\dagger} U_{\dagger}(y)\right)+\partial_{y} U_{\dagger}(y)\right) d y \\
& \quad+\sum_{ \pm} \pm\left.\left(y+L_{-}\right) U_{\dagger}(y) \partial_{y} w^{\prime}(y, \pm 0)\right|_{-L_{-}} ^{L_{+}-\varrho} \\
= & \sum_{ \pm} \pm \int_{-L_{-}}^{L_{+}-\varrho} w^{\prime}(y, \pm 0) \partial_{y} U_{\dagger}(y) d y+U_{\dagger}\left(L_{+}\right) L K \varrho^{-1 / 2}+O\left(\varrho^{1 / 2}\right) .
\end{aligned}
$$

Thus, the growing terms mutually cancel, and after the limit passage as $\varrho \rightarrow+0$ and $R \rightarrow+\infty$, we can continue the calculations (2.38), obtaining the same formula

$$
\begin{aligned}
b^{\prime} & =-\frac{1}{2} J_{\dagger}^{\prime \prime}\left(2\left(y+L_{-}\right)\right) \\
& :=-\frac{1}{2} \int_{-1 / 2}^{1 / 2} \int_{-\infty}^{+\infty}\left(\left|\partial_{y} w^{\prime}(y, z)\right|^{2}+\left|\partial_{z} w^{\prime}(y, z)\right|^{2}-\Lambda_{\dagger}\left|w^{\prime}(y, z)\right|^{2}\right) d y d z<0,
\end{aligned}
$$

as in Subsection 4 of $\S 2$. So, relations (2.32) and (2.42) survive completely.

The asymptotic justification process complicates somewhat because the term $w^{\prime \prime}(y, z)$ of the Ansatz (2.31) acquires the singularity $O\left(r_{+}^{-1 / 2}\right)$. This singularity is due to the shift of the screen vertex by the rule

$$
\left(-L_{-}+\left(1+\varepsilon^{2}\right)^{-1 / 2} L, \varepsilon\left(1+\varepsilon^{2}\right)^{-1 / 2} L\right) \mapsto\left(L_{+}, 0\right),
$$

when we pass to $\varepsilon=0$. Such "step" perturbations of domains are studied in detail: in [37, 38, 39] the necessary modifications of the global asymptotic approximation can be found, which makes is possible to eliminate the singularity in question. Otherwise, the 
justification procedure remains as in $\S 3$. Another approach to constructing and justifying asymptotic formulas is related to the initial coordinate change

$$
\begin{aligned}
& (y, z) \quad \mapsto \quad(\mathfrak{y}, \mathfrak{z}), \\
& \mathfrak{y}=\left(1-X_{\Theta}(y, z)\right) y+X_{\Theta}(y, z)\left(-L_{-}+\left(1+\varepsilon^{2}\right)^{1 / 2}\left(y+L_{-}\right)\right), \\
& \mathfrak{z}=\left(1-X_{\Theta}(y, z)\right) z+X_{\Theta}(y, z)\left(z-\varepsilon\left(1+\varepsilon^{2}\right)^{-1 / 2} L y\right),
\end{aligned}
$$

which takes the waveguide $\Theta^{\varepsilon}$ with inclined screen to the waveguide with screen orthogonal to the $z$-axis; in (4.14), $X_{\Theta}$ is a cutoff function supported on some neighborhood of the set $\Theta^{\varepsilon} \cup \Theta^{0}$ and equal to 1 in some smaller neighborhood. Since the map $(y, z) \mapsto(\mathfrak{y}, \mathfrak{z})$ differs little from the identity, the boundary value problem obtained from (1.5) -1.7) turns out to be a small regular perturbation of the limit problem (1.13), (1.14), i.e., the formal construction of the asymptotic expression can be done along the classical lines. However, the verification of inequality (4.13) becomes technically difficult and intricate: the differential operators $\mathfrak{L}_{1}$ and $\mathfrak{L}_{2}$ in the expansion

$$
\Delta_{(y, z)}=\Delta_{(\mathfrak{y}, \mathfrak{z})}+\varepsilon \mathfrak{L}_{1}\left(\mathfrak{y}, \mathfrak{z}, \nabla_{(\mathfrak{y}, \mathfrak{z})}\right)+\varepsilon^{2} \mathfrak{L}_{2}\left(\mathfrak{y}, \mathfrak{z}, \nabla_{(\mathfrak{y}, \mathfrak{z})}\right)+\cdots
$$

have variable compactly supported coefficients and are not formally selfadjoint.

Apparently, both approaches work in the case of many-dimensional waveguides with inclined screens, but the author has not studied this.

5. A screen touching the boundary. For a planar waveguide with a screening obstacle attached to the boundary of the half-strip (Figure 7d), the asymptotic formulas for eigenvalues and eigenfunctions can be found as this was done in $\S \S 2$ and 3 (see also Subsection $4, \S 5$ ), because, as before, the boundaries of domains have only corner points. Moreover, some of the phenomena discussed above disappear. For instance, unlike the "coin" in Figure 7a, the "tooth" in Figure 7d does not require consideration of boundary layers, despite the fact that condition (1.3) is violated. We consider this in more detail.

Let $\Omega=(-1 / 2,1 / 2) \times \mathbb{R}$ be a strip of unit width, and let the screen be given by the formula

$$
\Theta^{\varepsilon}=\left\{(y, z): y \in[l, 1 / 2],-\varepsilon h_{-}(y-l) \leq z \leq \varepsilon h_{+}(y-l)\right\},
$$

where $-1 / 2<l<1 / 2$ and $h=h_{-}+h_{+} \geq 0$. If $h>0$, so that the asymptotic Ansätze (2.15) and (2.25) can be taken, relation (2.22) remains the same: the calculations retain their validity, though for another reason, as compared to Subsection 2 of $\S 2$ : when integrating by parts, the term $\left.U_{\dagger}(y) H(y) \partial_{y} U_{\dagger}(y)\right|_{l} ^{1 / 2}$ vanishes because $H(y)=h(y-l)$ and $U_{\dagger}( \pm 1 / 2)=0$. Also, condition (2.24) takes the form

$$
J_{\dagger}(H)=\frac{h}{4}(1+\cos (2 \pi l))>0,
$$

and is fulfilled for all $l \in(-1 / 2,1 / 2)$ by (4.11), i.e., formulas (2.25) and (2.29) are true.

But if $h=0$ and $h_{+}=-h_{-} \neq 0$ (the "tooth" becomes one-dimensional), then, as in Subsection 4 of $\S 4$, the third term $w^{\prime \prime}(y, z)$ in (2.31) does not belong to $H_{\text {loc }}^{1}\left(\Omega^{0}\right)$. The point is that the second term $w^{\prime}$ is a solution of the Helmholtz equation with the boundary conditions

$$
w^{\prime}( \pm 1 / 2, z)=0, \quad z \in \mathbb{R}, \quad \mp \partial_{z} w^{\prime}(y, \pm 0)= \pm h_{+} \partial_{y} U_{\dagger}(y), \quad y \in(l, 1 / 2) .
$$

At the corner point $(1 / 2, \pm 0)$ the type of the boundary condition changes, and $w^{\prime}$ satisfies the equation

$$
w^{\prime}(y, z)=\chi_{+}(x) \sqrt{8} h_{ \pm} r_{+}\left(\ln r_{+} \cos \varphi_{+}+\left(\varphi_{+} \mp \pi / 2\right) \sin \varphi_{+}\right)+\widehat{w}^{\prime}(x)
$$

with a remainder term $\widehat{w}^{\prime}$ of class $H_{\mathrm{loc}}^{2}\left(\Omega^{0}\right)$. The factor on the right-hand side of (4.15) is found with the help of (4.11), and the form of the angular part is a consequence 


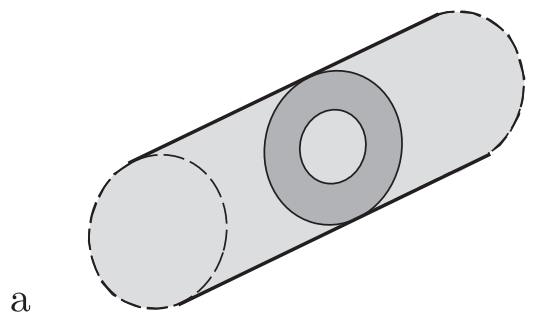

$\mathrm{b}$

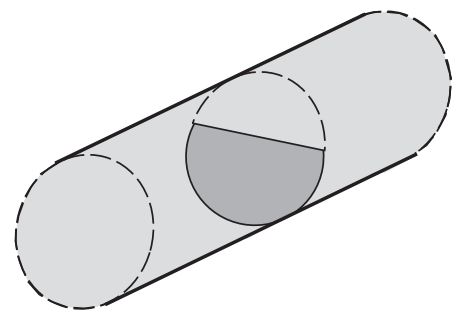

FIGURE 9

of a standard procedure (see, e.g., [25, Chapter 2]) that employs the polar coordinates centered at $(1 / 2,0)$. As a result, the right-hand side of the boundary condition (2.35) acquires a singularity of class $O\left(r_{+}^{-1}\right)$, which expels the solution from $H_{\text {loc }}^{1}\left(\Omega^{0}\right)$. The same tricks as in Subsection 4 of $\S 4$ allow us to fix the situation and to arrive at the usual relations (2.32) and (2.42). It should be mentioned that, under the singular perturbations of angles in boundary value problems for fourth order equations and systems, some logarithmic singularities of solutions, similar to those in (4.15), result in unexpected effects called paradoxes in mechanics (cf. [40, 41]), but no such effects are observed for the Helmholtz equation.

Generalization to many dimensions meets no difficulties only if the set $\Theta^{0}$ touches the surface along the entire set $\partial \theta \times\{0\}$ (Figure 9a). However, in the case of a half-closed cushion (Figure 9b) some new problems arise concerning the solutions' singularities at the points of the type of polytope vertices. Nevertheless, the exponents of such singularities are known (see [42, §3.8] and the references therein), so that the asymptotic formulas can be found as before. Finally, we note that, in accordance with the sufficient condition (1.24), (1.26) for the discrete spectrum to be nonempty for any nontrivial perturbation of the screens depicted in Figure 9 (see Subsection 2 of $\S 1$ ), if thickness is kept to be zero, then the discrete spectrum contains an eigenvalue $\lambda_{1}^{\varepsilon} \in\left(0, \Lambda_{\dagger}\right)$, and this eigenvalue is unique by Proposition 3.1 .

\section{REFERENCES}

[1] O. A. Ladyzhenskaya, Boundary value problems of mathematical physics, Nauka, Moscow, 1973; English transl., Appl. Math. Sci., vol. 49, Springer-Verlag, New York, 1985. MR 0599579 (58:29032) MR0793735 (87f:35001)

[2] S. G. Mikhlin, Linear partial differential equations, Vyssh. Shkola, Moscow, 1977. (Russian) MR0510535 (80a:35001)

[3] M. Sh. Birman and M. Z. Solomyak, Spectral theory of selfadjoint operators in Hilbert space, Leningrad. Gos. Univ., Leningrad, 1980; English transl., D. Reidel Publ. Co., Dordrecht, 1987. MR0609148 (82k:47001) MR1192782 (93g:47001)

[4] C. H. Wilcox, Scattering theory for diffraction gratings, Appl. Math. Sci., vol. 46, Springer, New York, 1984. MR0725334 (85f:78020)

[5] C. M. Linton and P. McIver, Embedded trapped modes in water waves and acoustics, Wave Motion 45 (2007), 16-29. MR 2441664 (2009e:35216)

[6] V. G. Maz'ya, S. A. Nazarov, and B. A. Plamenevskiǔ, Asymptotic expansions of eigenvalues of boundary value problems for the Laplace operator in domains with small openings, Izv. Akad. Nauk SSSR Ser. Mat. 48 (1984), no. 2, 347-371; English transl., Math. USSR-Izv. 24 (1985), no. 2, 321-346. MR0740795 (86b:35152)

[7] _ Asymptotische Theorie elliptischer Randwertaufgaben in singulär gestörten Gebieten. I, Math. Lehrbücher Monogr. II. Abt. Math. Monogr., Bd. 82, Akademie-Verlag, Berlin, 1991; English transl., V. Maz'ya, S. Nazarov, and B. Plamenevskij. Asymptotic theory of elliptic boundary value problems in singularly perturbed domains. Vol. 1, Oper. Theory Adv. Appl., vol. 111, Birkhäuser Verlag, Basel, 2000. MR1101139 (92g:35059) MR1779977 (2001e:35044a) 
[8] I. V. Kamotskiu and S. A. Nazarov, Elastic waves localized near periodic families of defects, Dokl. Akad. Nauk 368 (1999), no. 6, 771-773; English transl., Dokl. Phys. 44 (1999), no. 10, 715-717. MR:1749046(2001c:74043)

[9] Exponentially decreasing solutions of the problem of diffraction by a rigid periodic boundary, Mat. Zametki 73 (2003), no. 1, 138-140; English transl., Math. Notes 73 (2003), no. 1-2, 129-131. MR 1993547

[10] P. Duclos and P. Exner, Curvature-induced bound states in quantum waveguides in two and three dimensions, Rev. Math. Phys. 7 (1995), no. 1, 73-102. MR.1310767 (95m:81239)

[11] D. Borisov, P. Exner, R. Gadyl'shin, and D. Krejčiřik, Bound states in weakly deformed strips and layers, Ann. H. Poincaré 2 (2001), no. 3, 553-572. MR.1846856 (2002f:35053)

[12] R. Gadyl'shin, On regular and singular perturbations of acoustic and quantum waveguides, C. R. Mecanique 332 (2004), no. 3, 647-652.

[13] D. Borisov, Asymptotic behavior of the spectrum of a waveguide with distant perturbations, Math. Phys. Anal. Geom. 10 (2007), 155-196. MR2342630 (2009b:35049)

[14] P. Exner, P. Šeba, M. Tater, and D. Vaněk, Bound states and scattering in quantum waveguides coupled laterally through a boundary window, J. Math. Phys. 37 (1996), no. 10, 4867-4887. MR.1411612 (98b:81032)

[15] D. Borisov, P. Exner, and R. Gadyl'shin, Geometric coupling thresholds in a two-dimensional strip, J. Math. Phys. 43 (2002), 6265-6278. MR1939643(2004d:35189)

[16] D. I. Borisov, The discrete spectrum of a pair of asymmetric window-coupled waveguides, Mat. Sb. 197 (2006), no. 4, 3-32; English transl., Sb. Math. 197 (2006), no. 3-4, 475-501. MR2263787 (2007g:35162)

[17] I. V. Kamotskiǔ and S. A. Nazarov, Wood's anomalies and surface waves in the problem of scattering by a periodic boundary. I, Mat. Sb. 190 (1999), no. 1, 109-138; English transl., Sb. Math. 190 (1999), no. 1-2, 111-141. MR1700697 (2000j:35047)

[18] _ Wood's anomalies and surface waves in the problem of scattering by a periodic boundary. II, Mat. Sb. 190 (1999), no. 2, 43-70; English transl., Sb. Math. 190 (1999), no. 1-2, 205-231. MR 1701000 (2000j:35048)

[19] - Trapped modes, surface waves and Wood's anomalies for gently sloped periodic boundaries, C. R. Acad. Sci. Ser. 2328 (2000), 423-428.

[20] M. I. Vishik and L. A. Lyusternik, Regular degeneration and boundary layer for linear differential equations with small parameter, Uspekhi Mat. Nauk 12 (1957), no. 5, 3-122. (Russian) MR0096041 $(20: 2539)$

[21] V. A. Kondrat'ev, Boundary value problems for elliptic equations in domains with conical or angular points, Trudy Moskov. Mat. Obshch. 16 (1967), 209-318; English transl. in Trans. Moscow Math. Soc. 1967 (1968). MR0226187 (37:1777)

[22] V. G. Maz'ya and B. A. Plamenevskiu, The coefficients in the asymptotics of solutions of elliptic boundary value problems with conical points, Math. Nachr. 76 (1977), 29-60. (Russian) MR0601608 (58:29176

[23] Estimates in $L_{p}$ and in Hölder classes, and the Miranda-Agmon maximum principle for the solutions of elliptic boundary value problems in domains with singular points on the boundary, Math. Nachr. 81 (1978), 25-82. (Russian) MR0492821(58:11886)

[24] S. A. Nazarov, Polynomial property of selfadjoint elliptic boundary value problems and the algebraic description of their attributes, Uspekhi Mat. Nauk 54 (1999), no. 5, 77-142; English transl., Russian Math. Surveys 54 (1999), no. 5, 947-1014. MR.1741662(2001k:35073)

[25] S. A. Nazarov and B. A. Plamenevskiǔ, Elliptic problems in domains with piecewise smooth boundaries, Nauka, Moscow, 1991; English transl., de Gruyter Exp. in Math., vol. 13, Walter de Gruyter, Berlin, 1994. MR:1283387(95h:35001)

[26] V. A. Kozlov, V. G. Maz'ya, and J. Rossmann, Elliptic boundary value problems in domains with point singularities, Math. Surveys Monogr., vol. 52, Amer. Math. Soc., Providence, RI, 1997. MR 1469972 (98f:35038)

[27] S. A. Nazarov, Properties of spectra of boundary value problems in cylindrical and quasicylindrical domains, Sobolev Spaces in Mathematics. II (Maz'ya V., ed.), Int. Math. Ser. (N. Y.), vol. 9, Springer, New York, 2009, pp. 261-309. MR2484629(2009m:35508)

[28] V. A. Kondrat'ev, The smoothness of the solution of the Dirichlet problem for second order elliptic equations in a piecewise smooth domain, Differentsial'nye Uravneniya 6 (1970), no. 10, 1831-1843; English transl. in Differ. Equations 6 (1970). MR0282052(43:7766)

[29] V. G. Maz'ya and B. A. Plamenevskiu, $L_{p}$-estimates of solutions of elliptic boundary value problems in domains with ribs, Trudy Moskov. Mat. Obshch. 37 (1978), 49-93; English transl. in Trans. Moscow Math. Soc. 1980, no. 1. MR0514327 (81b:35027) 
[30] S. A. Nazarov and B. A. Plamenevskiŭ, The Neumann problem for selfadjoint elliptic systems in a domain with a piecewise-smooth boundary, Trudy Leningrad. Mat. Obshch. 1 (1990), 174-211; English transl., Amer. Math. Soc. Transl. (2), vol. 155, Amer. Math. Soc., Providence, RI, 1993, pp. 169-206. MR1104210(92e:35065)

[31] S. A. Nazarov and Yu. A. Romashev, Change of the intensity coefficient under destruction of a bridge between two collinear cracks, Izv. Akad. Nauk Armyan. SSR Ser. Mekh. 1982, no. 4, 30-40. (Russian)

[32] S. A. Nazarov, Asymptotic conditions at a point, selfadjoint extensions of operators, and the method of matched asymptotic expansions, Trudy S.-Peterburg. Mat. Obshch. 5 (1998), 112-183; English transl., Amer. Math. Soc. Transl. (2), vol. 193, Amer. Math. Soc.. Providence, RI, 1999, pp. 77-125. MR $1736907(2000 \mathrm{~m}: 35016)$

[33] A. M. Il'in, Matching of asymptotic expansions of solutions of boundary value problems, Nauka, Moscow, 1989; English transl., Transl. Math. Monogr., vol. 102, Amer. Math. Soc., Providence, RI, 1992. MR 1007834 (90i:35062), MR1182791 (93g: 35016)

[34] S. A. Nazarov and B. A. Plamenevskiŭ, Elliptic problems with radiation conditions on the edges of the boundary, Mat. Sb. 183 (1992), no. 10, 13-44; English transl., Russian Acad. Sci. Sb. Math. 77 (1994), no. 1, 149-176. MR1202790 (94b:35091)

[35] _ Selfadjoint elliptic problems: the scattering operator and the polarization operator on edges of the boundary, Algebra i Analiz 6 (1994), no. 4, 157-186; English transl., St. Petersburg Math. J. 6 (1995), no. 4, 839-863. MR1304098 (95j:35060)

[36] A. M. Il'in, A boundary value problem for an elliptic equation of second order in a domain with a narrow slit. I. The two-dimensional case, Mat. Sb. (N.S.) 99 (1976), no. 4, 514-537; English transl. in Math. USSR-Sb. 28 (1976), no. 4. MR0407439 (53:11214)

[37] S. A. Nazarov and M. V. Olyushin, Perturbations of eigenvalues of the Neumann problem due to variation of a domain's boundary, Algebra i Anliz 5 (1993), no. 2, 169-188; English transl., St. Petersburg Math. J. 5 (1994), no. 2, 371-387. MR.1223177 (94f:35020)

[38] S. A. Nazarov and M. Shpekovius-Nol̆gebauer, Application of the energy criterion of fracture for determining the shape of a slightly curved crack, Prikl. Mekh. Tekhn. Fiz. 47 (2006), no. 5, 119-130; English transl., J. Appl. Mech. Techn. Phys. 47 (2006), no. 5, 714-723. MR2258933 (2007d:74085)

[39] S. A. Nazarov, Asymptotic modeling of a problem with contrasting stiffness, Zap. Nauchn. Sem. S.-Peterburg. Otdel. Mat. Inst. Steklov. (POMI) 369 (2009), 164-201. (Russian) MR2749206

[40] V. G. Maz'ya and S. A. Nazarov, Paradoxes of the passage to the limit in solutions of boundary value problems for the approximation of smooth domains by polygons, Izv. Akad. Nauk SSSR Ser. Mat. 50 (1986), no. 6, 1156-1177; English transl., Math. USSR-Izv. 29 (1987), no. 3, 511-533. MR0883157 (88i:35016)

[41] S. A. Nazarov and M. V. Olyushin, Approximation of smooth contours by polygonal ones. Paradoxes in problems for the Lamé system, Izv. Ross. Akad. Nauk Ser. Mat. 61 (1997), no. 3, 159-186; English transl., Izv. Math. 61 (1997), no. 3, 619-646. MR1478563 (98h:73022)

[42] V. Z. Parton and P. I. Perlin, Methods of the mathematical theory of elasticity, Nauka, Moscow, 1981; French transl., Métodes de la théorie mathématique de l'élasticité, vols. 1, 2, Mir, Moscow, 1984. MR0617911(82i:73002) MR0776415(86b:73002a) MR0776416(86b:73002b)

Institute of Mechanical Engineering Problems, Bol'shoĭ Prospekt V. O. 61, St. Petersburg 199178, Russia

E-mail address: srgnazarov@yahoo.co.uk

Received 25/JAN/2010

Translated by A. PLOTKIN 